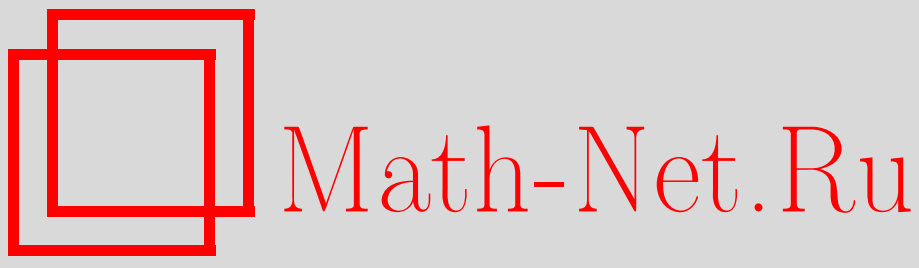

А. М. Шур, Структура множества бескубных $Z$-слов в двухбуквенном алфавите, Изв. РАН. Сер. матем., 2000, том 64, выпуск 4, 201-224

DOI: https://doi.org/10.4213/im301

Использование Общероссийского математического портала Math-Net.Ru подразумевает, что вы прочитали и согласны с пользовательским соглашением

http: //www. mathnet.ru/rus/agreement

Параметры загрузки:

IP : 52.87 .193 .239

26 апреля 2023 г., 18:18:08 
УДК 512.532.2+519.11

\author{
A. М. Шур
}

\title{
Структура множества бескубных $Z$-слов в двухбуквенном алфавите
}

\begin{abstract}
Объектом изучения являются $Z$-слова, т.е. бесконечные цепочки алфавитных символов, индексированные целыми числами. Рассматривается вполне упорядоченное семейство подмножеств множества всех бескубных $Z$-слов в двухбуквенном алфавите, построенное на основе вводимого понятия локальной экспоненты $Z$-слова. Решен вопрос о существовании бескубных $Z$-слов заданной локальной экспоненты. Описана комбинаторная структура множества всех сильно бескубных $Z$-слов, являющихся $Z$-словами локальной экспоненты 2 , минимальной из возможных. Указано важное отличие сильно бескубных $Z$-слов от $Z$-слов большей локальной экспоненты.

Библиограбия: 12 наименований.
\end{abstract}

\section{Введение}

$Z$-слова, т.е. бесконечные цепочки символов, индексированные целыми числами, наряду с $\omega$-словами - бесконечным цепочками, индексированными натуральными числами, представляют собой наиболее естественные обобщения обычных (конечных) слов. Отметим, что ряд пионерских результатов в комбинаторной теории слов связан именно с бесконечными словами (см., например, [1]-[3]).

В исследовании бесконечных слов можно выделить два основных подхода. Первый из них состоит в рассмотрении для бесконечных слов задач, уже ставившихся для конечных слов (при необходимости адаптируя соответствующие постановки). Таким образом, можно выяснить, какие свойства обычных слов сохраняются при переходе к бесконечным, а какие видоизменяются, теряются либо заменяются новыми. В качестве примера отметим работу [4], в которой показано, что очень многие понятия из обычной теории языков могут быть обобщены на множества $\omega$-слов. При этом обобщения понятий рационального языка и конечного автомата могут быть выбраны таким образом, чтобы для них выполнялся аналог теоремы Клини.

Второй подход состоит в изучении свойств, специфических для бесконечных объектов. В этом направлении отметим монографиию [5], одна из глав которой посвящена описанию топологических свойств множества всех бинарных сильно бескубных $Z$-слов относительно естественно определенной метрики.

Данная работа находится в рамках первого подхода: все основные понятия применимы и к конечным словам. Исследуемые свойства и методы исследования являются комбинаторными.

Объектом нашего изучения является множество всех бескубных $Z$-слов в двухбуквенном алфавите. Куб является минимальной степенью слова, избегаемой в минимальном нетривиальном алфавите, что обусловило высокий интерес к проблемам, связанным со свойством бескубности. О структуре множества конечных

$$
\text { (C) А. М. Шур, } 2000
$$


бескубных слов известно крайне мало. Это множество велико (количество бескубных слов длины $n$ зависит от $n$ экспоненциально, см. [6]); хорошо изучено лишь небольшое его подмножество, состоящее из всех сильно бескубных слов (количество которых зависит от длины менее чем квадратично; см. [7]). При рассмотрении $Z$-слов мы имеем аналогичную картину: практически ничего не известно о множестве всех бескубных $Z$-слов, но есть существенная информация о множестве всех сильно бескубных $Z$-слов (см., например, [5], [8]).

Автору удалось получить важную информацию об устройстве множества всех бескубных $Z$-слов. В работе введено понятие экспоненты, избегаемой словом $(Z$-словом $)$, на основе которого определена иерархия подмножеств указанного множества. Доказан ряд свойств иерархии в целом и наиболее важных ее ступеней.

Текст состоит из трех параграфов. В первом из них приводятся необходимые определения и вспомогательные комбинаторные утверждения, а также вводится иерархия множеств бескубных слов. В $§ 2$ приведено описание множества сильно бескубных $Z$-слов (первой ступени упомянутой иерархии) в комбинаторных терминах; $\S 3$ содержит сведения о мощности данной иерархии, ее устройстве и важное свойство второй ступени этой иерархии.

\section{$\S 1$. Определения и предварительные замечания}

\section{1. Определения.}

1. Пусть $A$-конечный алфавит. Кроме слов, т.е. конечных последовательностей алфавитных символов, определим также три типа счетных последовательностей таких символов. А именно, последовательности вида

$$
\begin{gathered}
a_{1} a_{2} \ldots a_{n} \ldots \\
\ldots a_{-n} \ldots a_{-2} a_{-1} a_{0} \\
\ldots a_{-n} \ldots a_{-2} a_{-1} a_{0} a_{1} a_{2} \ldots a_{n} \ldots
\end{gathered}
$$

мы называем соответственно $\omega$-, $\omega^{*}$ - и $Z$-словами. Для обозначения $\omega$-слов (соответственно $\omega^{*}$-слов) будем использовать те же буквы, что и для слов, но с индексом $\infty$ справа (соответственно слева). $Z$-слова будем обозначать жирными буквами.

Через $A^{+}\left(A^{\omega}, A^{Z}\right)$ обозначается множество всех непустых слов (соответственно всех $\omega$-слов, всех $Z$-слов) над алфавитом $A$. Пустое слово мы обозначаем через $\lambda$. Языками мы будем называть произвольные подмножества из $A^{+}, A^{\omega}, A^{Z}$ (из контекста всегда будет ясно, о множестве каких слов идет речь).

Ниже везде считается, что $A=\{a, b\}$.

2. Слово $V$ называется подсловом слова $W$ (или говорят, что $W$ coдержит $V$, обозначается $V \leqslant W)$, если $W=P V Q$ для некоторых слов $P, Q$. При этом $V$ называется префиксом (суффиксом) $W$, если $P=\lambda$ (соответственно $Q=\lambda$ ). Непустой префикс (суффикс) слова называется собственнылм, если он не совпадает с самим словом.

3. Через $\phi$ будем обозначать эндоморфизм $A^{+}$, определяемьй равенствами

$$
\phi(a)=a b, \quad \phi(b)=b a .
$$

Слова

$$
U_{n}=\phi^{n}(a), \quad V_{n}=\phi^{n}(b), \quad n \geqslant 0,
$$


назьваются словами Туэ-Морса.

Справедливы равенства

$$
U_{n+1}=U_{n} V_{n}, \quad V_{n+1}=V_{n} U_{n}
$$

Так как $U_{n}$ является собственным префиксом $U_{n+1}$, а $V_{n}$ - собственным префиксом $V_{n+1}$, то сушествуют $\omega$-слова $U_{\infty}, V_{\infty}$, являющиеся "пределами" последовательностей $\left\{U_{n}\right\}$ и $\left\{V_{n}\right\}$ соответственно. Определим также $\omega^{*}$-слова ${ }_{\infty} U, \infty V$, получаюшиеся "переворачиванием" $\omega$-слов $U_{\infty}$ и $V_{\infty}$ соответственно.

Слова $U_{n}$ и $V_{n}$ будем называть также $n$-иелочками.

4. Определим ряд комбинаторных параметров, связанных с произвольным словом $W$. Длина слова $W$ обозначается через $|W|$. Мы считаем, что буквы в слове $W$ занумерованы по порядку от 1 до $|W|$ (слева направо). Номер, соответствующий каждой букве, входящей в $W$, мы называем ее позицией в $W$. Буквы в бесконечных словах занумерованы от 1 до $\infty$ ( $\omega$-слова), от $-\infty$ до $0\left(\omega^{*}\right.$-слова) и от $-\infty$ до $\infty$ $\left(Z\right.$-слова). Через $0_{W}, 1_{W}$ будем обозначать первую (соответственно последнюю) букву слова $W$. Запись $W_{[i]}$ означает $i$-ю букву слова (бесконечного слова) $W$.

Число $p>0$ называется периодом слова $W$, если $W_{[i]}=W_{[i+p]}$ для всякого $i$ такого, что $1 \leqslant i \leqslant|W|-p$. В частности, по определению слово $W$ имеет период $|W|$. Минимальный период $W$ обозначается через $\operatorname{per}(W)$.

При $n \geqslant 2$ выполняются равенства $\operatorname{per}\left(U_{n}\right)=\operatorname{per}\left(V_{n}\right)=3 \cdot 2^{n-2}$.

Как и в работе [9], әкспонентой слова $W$ мы называем число $\exp (W)=$ $|W| / \operatorname{per}(W)$. Из определения видно, что целую экспоненту, большую единищы, имеют в точности слова, являюшиеся целыми степенями других слов. Таким образом, экспонента является обобщением степени на нецелые показатели.

Следуюшее определение играет ключевую роль в данной работе. Локальной әкспонентой слова $W$ мы будем называть число $\operatorname{lexp}(W)=\max \{\exp (V)$ $V \leqslant W\}$.

Локальная әкспонента бесконечного слова определяется так же, только максимум в формуле необходимо заменить на супремум.

5. Разбиением $W$ будем называть произвольное представление $W$ в виде произведения сомножителей. Сомножители - элементы разбиения - будем называть блоками. Разбиение бесконечного слова определяется точно так же.

Слово $W$ будем называть равномернымм, если оно допускает разбиение вида

$$
W=c Q_{1} \ldots Q_{n} d
$$

где $c, d \in\{\lambda, a, b\}$, а все $Q_{i}$ являются 1-цепочками.

Бесконечное слово называется равномерным, если всякое его подслово равномерно.

Из определения слов Туэ-Морса, очевидно, следует, что все они равномерны.

6 . Говорят, что слово $W$ избегает слово $V$, если $W$ не содержит образов $V$ при гомоморфизмах свободных полугрупп. Слово $V$ называется избегаемылм в алфавите $A$, если в $A^{+}$найдется бесконечно много слов, избегающих $V$.

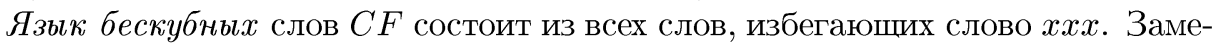
тим, что $C F=\{W \mid \operatorname{lexp}(W)<3\}$.

Язык сильно бескубных слов $O F$ состоит из всех слов, избегаюших слова $x x x$ и $x y x y x$. Можно проверить, что $O F=\{W \mid \operatorname{lexp}(W) \leqslant 2\}$.

Язык Туэ-Морса $T M$ состоит из всех слов Туэ-Морса и всех их подслов. 
Включение $T M \subseteq O F$ было впервые доказано А. Туэ (см. [1]). Это включение строгое: так, например, aаbaa $\in O F \backslash T M$. Очевидно также, что $O F \subset C F$.

Через $L^{Z}$ мы обозначаем язык, состояший из всех $Z$-слов, все конечные подслова которых принадлежат языку $L$.

1.2. Сохранение экспонент при действии морфизма $\phi$. Определенное выше отображение $\phi$ (морфизм Туэ-Морса) обладает множеством примечательных свойств. Как показывают два следующих предложения, $\phi$ сохраняет экспоненту и локальную экспоненту слова в максимально широком диапазоне, в принципе возможном для несюръективных отображений. Свойство сохранения экспонент при действии $\phi$ нам потребуется при доказательстве большинства результатов работы.

ПРЕДЛОЖЕНИЕ 1.1. Пусть $W \in A^{+} u \exp (W)>1$. Тогдa

$$
\exp (\phi(W))=\exp (W) .
$$

ДоКАЗАТЕЛЬСТво. Положим $p=\operatorname{per}(W)$. Тогда, очевидно, слово $\phi(W)$ имеет период $2 p$; значит, $\exp (\phi(W)) \geqslant \exp (W)$. Предположим, что у $\phi(W)$ есть также период $t<2 p$. Если число $t$ четное, то слово $W$ имеет период $t / 2$, меньший $p$; противоречие с минимальностью $p$. Следовательно, $t$ нечетное. Очевидно, $t \geqslant 3$.

Представим $\phi(W)$ в виде произведения $P Q$, где $|P|=t$. Покажем, что слово $Q$ не содержит подслов $a a$ и $b b$. В самом деле, пусть $a a \leqslant Q$. Тогда на $t$ позиций левее в слове $\phi(W)$ также находится подслово $a а$ (по определению периода). Так как $t$ нечетно, то одно из этих подслов начинается в $\phi(W)$ с нечетной позиции. Получаем противоречие, так как подслово длины 2 , начинающееся в $\phi(W)$ с нечетной позиции, должно быть 1-цепочкой.

Итак, $Q$ состоит из чередующихся букв. Таким образом, первая и $(2 p-t+1)$-я буквы слова $Q$ различны. Но эти две буквы суть $(t+1)$-я и $(2 p+1)$-я буквы в $\phi(W)$, которые совпадают по определению периода. Полученное противоречие доказывает, что у слова $\phi(W)$ нет периодов, меньших $2 p$, откуда следует требуемое утверждение.

ПРЕДЛОЖЕНИЕ 1.2. Пусть $W \in A^{+} u \operatorname{lexp}(W) \geqslant 2$. Тогда

$$
\operatorname{lexp}(\phi(W))=\operatorname{lexp}(W)
$$

ДокАЗАТЕльСтво. Положим $\beta=\operatorname{lexp}(W)$. Неравенство $\operatorname{lexp}(\phi(W)) \geqslant \beta$, очевидно, следует из определений $\phi$ и локальной экспоненты. Докажем, что при $\beta \geqslant 2$ справедливо и противоположное неравенство.

Предположим, что противоположное неравенство нарушается; выберем слово $W$ такое, что $\operatorname{lexp}(\phi(W))>\operatorname{lexp}(W)=\beta \geqslant 2$. Пусть $V$ - максимальное подслово в $\phi(W)$ экспоненты, большей $\beta$. Рассмотрим различные варианты расположения $V$ в слове $\phi(W)$. Через $V^{\prime}$ при этом будем обозначать максимальное подслово в $V$, начинающееся в $\phi(W)$ с нечетной позиции и заканчивающееся на четной.

Если $V=V^{\prime}$, то слово $\phi^{-1}(V)$ содержится в $W$. По предложению 1.1 экспонента последнего слова равна экспоненте $V$. Противоречие с условием $\operatorname{lexp}(W)=\beta$.

Пусть $V=V^{\prime} c$, где $c \in A$, и $p=\operatorname{per}(V)$. Из предложения 1.1 следует, что $\exp \left(V^{\prime}\right) \leqslant \beta$, т.е. $\exp (V)>\exp \left(V^{\prime}\right)$ и, значит, $\operatorname{per}(V)=\operatorname{per}\left(V^{\prime}\right)$. Минимальньй период слова $V^{\prime}$ четен по предложению 1.1. Таким образом, слово $V c=V^{\prime} c c$ не 
имеет периода $p$, так как слово $V^{\prime}$ не может содержать подслово $c c$, начинающееся с нечетной позиции. Тогда слово $V d$, где $d \in A, d \neq c$, имеет период $p$. Следовательно, $\exp (V d)>\exp (V)$; осталось заметить, что $V d \leqslant \phi(W)$. Противоречие с максимальностью $V$.

Случай $V=c V^{\prime} \quad(c \in A)$ симметричен только что разобранному. В последнем оставшемся случае $\left(V=c V^{\prime} d\right)$ заметим, что пара условий: $\exp (V)>\exp \left(V^{\prime}\right)$ и $\exp (V)>2$, влечет за собой совпадение минимальных периодов слов $V$ и $V^{\prime}$. Далее проводим то же рассуждение, что и в предыдущем абзаце. Тем самым доказательство предложения завершено.

1.3. Иерархия множеств бескубных $Z$-слов. Скажем, что слово $W$ избегает экспоненту $\beta$, если $\operatorname{lexp}(W)<\beta$; соответственно экспоненту $\beta$ назовем избегаемой в $A$, если в $A^{+}$найдется бесконечное множество слов с локальной экспонентой, меньшей $\beta$. Данные определения согласуются со введенными ранее: слово $W$ избегает экспоненту $k$ в тех же случаях, когда оно избегает слово $x^{k}$.

Куб является минимальной степенью буквы, избегаемой в алфавите $A$; в то же время любая экспонента, строго большая двух, также избегаема в $A$ (бесконечный язык $O F$ состоит только из слов с локальной экспонентой, не превосходящей 2). Таким образом, точная числовая граница избегаемости в $A$ - это число 2 , а не 3 .

Определим два однопараметрических семейства языков следующим образом:

$$
\begin{aligned}
& L_{\beta}=\left\{W \in A^{+} \mid \operatorname{lexp}(W)<\beta\right\}, \\
& L_{\beta}^{\prime}=\left\{W \in A^{+} \mid \operatorname{lexp}(W) \leqslant \beta\right\},
\end{aligned}
$$

где $2 \leqslant \beta<3$. Как каждое семейство по отдельности, так и оба они в совокупности образуют вполне упорядоченные множества (цепи) по включению. При этом, если судить по результатам исследований бескубных и сильно бескубных слов, чем выше язык находится в такой цепи, тем менее регулярно его внутреннее устройство.

Нас интересуют те свойства языков (по-видимому, более фундаментальные), которые переносятся на множества бесконечных слов (а именно, $Z$-слов). Такие свойства не зависят от "краевых эффектов" - влияния границ слова. Приведем такой пример. Еще в 1964 г. Готшалк и Хэдлунд доказали [8], что $O F^{Z}=T M^{Z}$. Далее, в работе [10] доказано, что большое подслово любого конечного сильно бескубного слова (составляющее не менее 7/10 от длины слова) принадлежит языку $T$ М. Таким образом, "настоящие" сильно бескубные слова - это в точности подслова слов Туэ-Морса.

Рассмотрим множество языков вида $L_{\beta}^{Z}, L_{\beta}^{\prime Z}$, где $2 \leqslant \beta<3$. Так же как и их конечные аналоги, эти языки образуют вполне упорядоченные множества. Вполне упорядоченное множество, состояшее из всех упомянутых языков из $A^{Z}$, мы будем называть иерархией множеств бескубных $Z$-слов. Свойствам иерархии в целом и ее отдельных ступеней и посвящена основная часть данной работы.

Рассмотрим отображения $F, F^{\prime}$ из $\mathbb{R}$ во множество всех языков из $A^{Z}$, определенные следующим образом:

$$
F(\beta)=L_{\beta}^{Z}, \quad F^{\prime}(\beta)=L_{\beta}^{\prime Z}
$$

Очевидно, что $F(\beta)=\varnothing$ при $\beta<2, F^{\prime}(\beta)=\varnothing$ при $\beta \leqslant 2$. Таким образом, исследование отображений $F, F^{\prime}$ имеет смысл на промежутке $[2,+\infty)$. Вначале 
покажем, что отображение $F$ непрерьвно слева. В самом деле, пусть последовательность $\left\{\beta_{i}\right\}$ сходится к числу $\beta$ снизу. Тогда

$$
\lim _{i \rightarrow \infty} F\left(\beta_{i}\right)=\bigcup_{i=1}^{\infty} L_{\beta_{i}}^{Z}=L_{\beta}^{Z}=F(\beta)
$$

что и означает непрерывность $F$ слева. Аналогичная проверка $F$ на непрерывность справа дает

$$
\lim _{i \rightarrow \infty} F\left(\beta_{i}\right)=\bigcap_{i=1}^{\infty} L_{\beta_{i}}^{Z}=L_{\beta}^{\prime Z}=F^{\prime}(\beta),
$$

т.е. непрерьвность $F$ в точке $\beta$ справа имеет место тогда и только тогда, когда $F(\beta)=F^{\prime}(\beta)$. Точно так же доказывается, что отображение $F^{\prime}(\beta)$ непрерывно справа, а непрерывным слева является в тех и только тех точках $\beta$, где $F(\beta)=$ $F^{\prime}(\beta)$.

Результаты, которые будут доказаны в $\S 3$, позволяют описать поведение обоих отображений на отрезке $[2,3]$. А именно, оба отображения являются константами вплоть до точки $\beta=7 / 3$, после чего их поведение меняется коренным образом: при $\beta \geqslant 7 / 3$ оба отображения становятся строго возрастаюшими и имеют скачок в каждой точке. При этом несложная экстраполящия доказательств из $\S 3$ показывает, что два последних свойства выполняются на всем промежутке $[7 / 3,+\infty)$.

Так как множества $\left\{L_{\beta}^{Z}\right\}_{\beta \in \mathbb{R}}, \quad\left\{L_{\beta}^{\prime Z}\right\}_{\beta \in \mathbb{R}}$ вполне упорядочены, то отображения $F$ и $F^{\prime}$ можно проиллюстрировать следующими графиками:
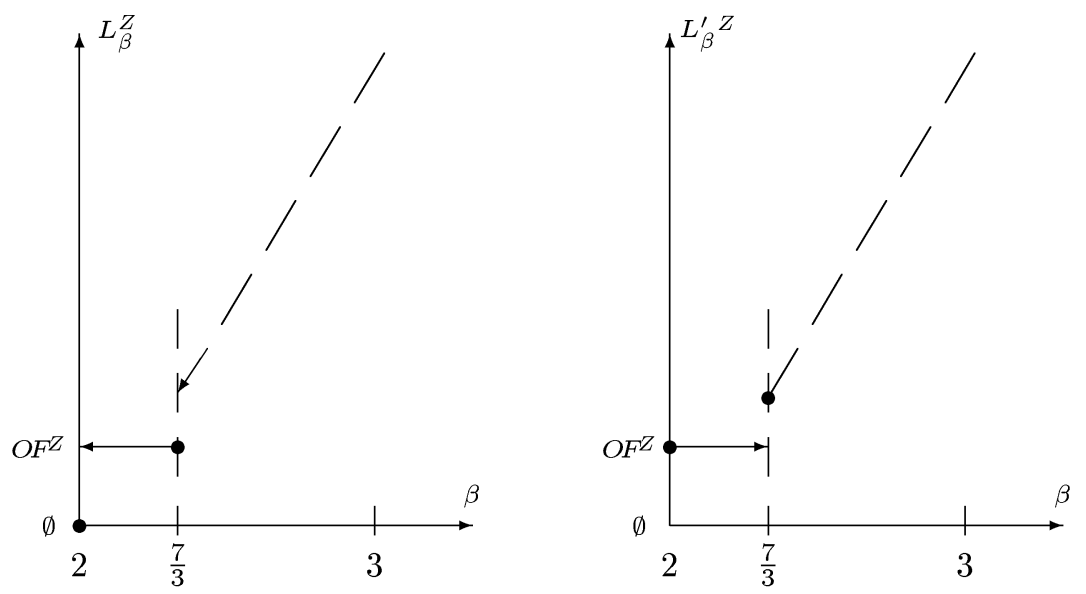

Из приведенных графиков видно, что во введенной иерархии есть две выделенные ступени. Первую из них занимает наименьший нетривиальный язык в иерархии - язык $O F^{Z}=L_{2}^{\prime Z}$, а вторую - следуюший за ним язык $L_{\alpha}^{\prime Z}$, где $\alpha=7 / 3$. Эти два языка изучаются в данной работе отдельно. 


\section{§2. Структура языка $O F^{Z}$}

Произвольное $Z$-слово мы будем представлять себе как числовую прямую, на которой "расставлены” буквы алфавита: каждому отрезку $[i-1, i]$, где $i$ - целое число, поставлена в соответствие $i$-я буква $Z$-слова. Определим элементарные операции над $Z$-словами: операция автоморфизма Aut (замена всех букв $a$ на $b$ и наоборот), операция реверса Rev (симметричное отражение $Z$-слова относительно точки 0) и набор операций сдвига Shift $k, k$ целое (сдвиг всего $Z$-слова на $k$ единищ вправо по числовой прямой). Два $Z$-слова будем называть изоморфными, если одно из них можно получить из другого с помощью элементарных операций.

В связи с уже упоминавшимся результатом [8] о равенстве языков $O F^{Z}$ и $T M^{Z}$ естественно возникает предположение, что различных (неизоморфных) сильно бескубных $Z$-слов немного и их достаточно несложно перечислить. Однако в [5] доказано, что таких $Z$-слов континуум. В данном параграфе мы дадим исчерпьваюшую комбинаторную характеризацию множества $O F^{Z}$, а именно перечислим все его элементы с точностью до изоморфизма.

2.1. Понятие $n$-разбиения. Порядок точки. Пусть дано $Z$-слово W. Разбиение $\mathbf{W}$ на $n$-цепочки (если такое сушествует) будем называть $n$-разбиением (тривиальное 0-разбиение существует для всякого $Z$-слова). Будем называть целочисленную точку $x$ әраничной для данного разбиения, если ближайшие к ней буквы $\mathbf{W}$ принадлежат разным блокам. Порядком точки $x$ относительно $\mathbf{W}$ $\left(\operatorname{deg}_{\mathbf{W}}(x)\right)$ будем называть максимум из чисел $n$ таких, что $x$ является граничной для некоторого $n$-разбиения $\mathbf{W}$, либо $\infty$, если максимум не сушествует. Очевидно, что для произвольной точки $x$ условие $\operatorname{deg}_{\mathbf{W}}(x) \geqslant n$ выполнено тогда и только тогда, когда $\operatorname{deg}_{\mathbf{W}}\left(x+k \cdot 2^{n}\right) \geqslant n$ для любого $k$.

ПРЕДЛОЖЕНИЕ 2.1. Для любого $\mathbf{W} \in O F^{Z}$ и любого натурального $n$ существует, и притом единственное, п-разбиение $\mathbf{W}$.

ДокАЗАтЕльство. Всякое подслово из $\mathbf{W}$ является элементом $T$. С. Седовательно, $\mathbf{W}$ равномерно, т.е. допускает разбиение на 1-цепочки.

Теперь рассмотрим $Z$-слово $\mathbf{W}^{\prime}$, получаемое из $\mathbf{W}$ операцией $\phi^{-1}$ : каждому блоку $Q_{i}$ построенного разбиения $\mathbf{W}$ соответствует буква $\phi^{-1}\left(Q_{i}\right)$ в $\mathbf{W}^{\prime}$. (Для однозначности определения потребуем, чтобы первой букве $\mathbf{W}^{\prime}$ соответствовал блок, содержаший первую букву W.) По предложению $1.1 \mathbf{W}^{\prime} \in O F^{Z}$; по доказанному выше сушествует 1-разбиение $\mathbf{W}^{\prime}$, которое в свою очередь индуцирует 2 -разбиение $\mathbf{W}$. Рассуждая по индукции, получаем, что существует $n$-разбиение $\mathbf{W}$ для всех $n$.

Предположим, что для некоторого $n$ существуют два $n$-разбиения $\mathbf{W}$. Рассмотрим $\omega$-подслово $\mathbf{W}$, начинающееся с граничной точки одного из разбиений двумя одинаковыми $n$-цепочками:

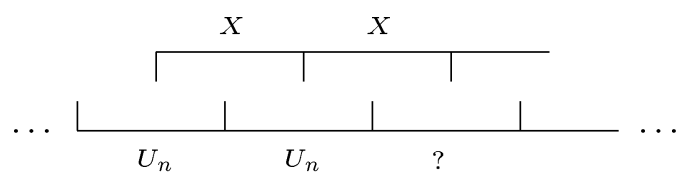

Как видно из рисунка, следующий за $U_{n} U_{n}$ блок выбранного разбиения начинается с той же буквы, что и $U_{n}$ (т.е. равен ему). Противоречие с сильной бескубностью W. Предложение доказано. 
СлеДСТВИЕ 2.2. Пусть $\mathbf{W} \in O F^{Z}, x-$ точка порядка $\geqslant n u k<2^{n}$. Тогда $\operatorname{deg}_{\mathbf{W}}(x+k)<n$.

ДокАЗАТЕЛЬСТво. Предположим, что $\operatorname{deg}_{\mathbf{W}}(x+k) \geqslant n$. Согласно определению порядка точки $Z$-слово $\mathbf{W}$ допускает два $n$-разбиения. Противоречие с предложением 2.1.

СлЕДСТвиЕ 2.3. Пусть $\mathbf{W} \in O F^{Z}$. Тогда из двух соседних точек поряд$\kappa a \geqslant n$ одна имеет порядок $n$, а другая - порядок $>n$.

ДоКАЗАТЕЛЬСТВо. Все точки, отстоящие от выбранных на расстояние, меньшее $2^{n}$, имеют порядок, меньший $n$ (следствие 2.2). Таким образом, ровно одна из выбранных точек будет являться граничной для $(n+1)$-разбиения $\mathbf{W}$.

СледСТВИЕ 2.4. $Z$-слово $\mathbf{W} \in O F^{Z}$ имеет не более одной точки поряд$\kappa a \infty$.

ДокАЗАТЕЛЬСтво. С учетом следствия 2.3 доказательство очевидно.

$Z$-слово $\mathbf{W}$ будем назьвать $Z$-словом бесконечного (конечного) порядка, если $\mathbf{W}$ имеет (соответственно не имеет) точку бесконечного порядка.

ПредЛОЖЕнИЕ 2.5. Язык $O F^{Z}$ содержит в точности два неизоморфных $Z$-слова бесконечного порядка.

ДокАЗАТЕЛЬСТво. Наличие в $Z$-слове точки порядка $\infty$ означает, что для любого $n \geqslant 0$ отрезок этого слова длины $2^{n}$, примыкающий справа или слева к этой точке, является $n$-цепочкой. Таким образом, справа от этой точки стоит по определению $\omega$-слово $U_{\infty}$ или $V_{\infty}$, а слева $-\omega^{*}$-слово $\infty U$ или $\infty V$. Перенесем точку порядка $\infty$ нашего $Z$-слова в 0 с помошью подходящей операции Shift и заметим, что

$$
\begin{aligned}
& \operatorname{Aut}\left({ }_{\infty} U U_{\infty}\right)=\infty V V_{\infty}, \\
& \operatorname{Aut}\left({ }_{\infty} U V_{\infty}\right)=\operatorname{Rev}\left({ }_{\infty} U V_{\infty}\right)={ }_{\infty} V U_{\infty},
\end{aligned}
$$

а из $\infty U U_{\infty}$ нельзя получить $\infty U V_{\infty}$ с помощью элементарных операций. Доказательство завершено.

2.2. Метод раскачивания. Построим пример сильно бескубного $Z$-слова конечного порядка. Предлагаемый метод построения будем называть методом раскачивания.

Возьмем числовую прямую и начнем расставлять на ней буквы. Пусть $x_{0}=$ $1 / 3, W_{0}=a=\mathbf{W}_{[1]}$.

$i$-й шАГ: к имеюшемуся слову $W_{i-1}$ приписываем $(i-1)$-цепочку, не совпадающую с $W_{i-1}$, с того конца, который ближе к $x_{0}$. Полученная $i$-цепочка и будет являться словом $W_{i}$.

Таким образом,

$$
\begin{aligned}
& W_{1}=\mathbf{W}_{[0]} \mathbf{W}_{[1]}=b a, \\
& W_{2}=\mathbf{W}_{[0]} \mathbf{W}_{[1]} \mathbf{W}_{[2]} \mathbf{W}_{[4]}=b a a b, \\
& W_{3}=\mathbf{W}_{[-4]} \cdots \mathbf{W}_{[4]}=a b b a b a a b \text { и т.д. }
\end{aligned}
$$

В пределе результатом описанного процесса будет $Z$-слово $\mathbf{W}$, каждое подслово которого лежит в некотором $W_{i}$, т.е. принадлежит $T M$. Значит, мы действительно построили элемент $O F^{Z}$. 
Точка, являющаяся серединой отрезка, занимаемого $W_{i}(i>0)$, имеет по построению порядок $n=i-1$. Докажем это по индукции. Точка $x=0$, являющаяся серединой отрезка, занятого $W_{1}$, имеем порядок 0 , так как отрезок длины 2 , находящийся справа от нее, равен $a a$.

ШАГ индукции: пусть $x$ и $y$ - середины отрезков, занимаемых $W_{i}$ и $W_{i+1}$ соответственно. Тогда $|x-y|=2^{i-1}$, т.е. $x$ и $y$ суть соседние точки порядка $\geqslant 2^{i-1}$ (по предположению индукции). По следствию $2.3 \operatorname{deg}_{\mathbf{w}}(y) \geqslant i$. Так как при построении $W_{i+2}$ рядом с $y$ оказываются две одинаковые $i$-цепочки (это две средние $i$-цепочки в $(i+2)$-цепочке), то окончательно $\operatorname{deg}_{\mathbf{W}}(y)=i$.

Середина отрезка, занимаемого $W_{i}$, находится на расстоянии $2^{n} / 3$ от точки $x_{0}$, а значит, является ближайшей к $x_{0}$ точкой порядка $\geqslant n$ по следствию 2.2 . В итоге имеем

$$
\forall k, \forall x \quad\left(\operatorname{deg}_{\mathbf{W}}(x) \geqslant k\right) \Rightarrow\left(\left|x-x_{0}\right| \geqslant \frac{2^{k}}{3}\right) .
$$

Следовательно, $Z$-слово $\mathbf{W}$ не имеет точки порядка $\infty$.

2.3. Теорема о $Z$-словах конечного порядка. Произвольному $Z$-слову $\mathbf{U} \in O F^{Z}$ поставим в соответствие две последовательности $\left\{y_{n}\right\}_{0}^{\infty}$ и $\left\{r_{n}\right\}_{0}^{\infty}$ по следующим правилам. Ближайшая к $x_{0}=1 / 3$ точка порядка $\geqslant n$ относительно $\mathbf{U}$ есть $y_{n}$, a $r_{n}=\left|x_{0}-y_{n}\right|$. Для всякого $n$ реализуется одна из двух возможностей:

1) $\operatorname{deg}_{\mathbf{U}}\left(y_{n}\right) \geqslant n+1$, тогда $y_{n+1}=y_{n}$ и соответственно $r_{n+1}=r_{n}$;

2) $\operatorname{deg}_{\mathbf{U}}\left(y_{n}\right)=n$, тогда по следствию 2.3 точки, отстоящие от $y_{n}$ на расстояние $2^{n}$, имеют порядок $\geqslant n+1$; таким образом, одна из этих точек есть $y_{n+1}$, т.е. $\left|y_{n+1}-y_{n}\right|=2^{n}$. Так как $r_{k}<2^{k-1}$ для любого $k$ (неравенство строгое, так как $r_{k}$ - не целое число), то $r_{n+1}=2^{n}-r_{n}$.

Таким образом, $\left\{r_{n}\right\}$ однозначно кодируется бинарной последовательностью $\left\{h_{n}\right\}_{0}^{\infty}$ по правилу

$$
\begin{gathered}
r_{n+1}=2^{n}-r_{n} \Rightarrow h_{n}=1 \\
r_{n+1}=r_{n} \Rightarrow h_{n}=0
\end{gathered}
$$

(можно записать $h_{n}=\operatorname{sgn}\left|r_{n+1}-r_{n}\right|$ ). Будем называть $\left\{h_{n}\right\}$ xapaктеристической последовательностью $\mathrm{U}$ и обозначать $\left\{h_{n}^{\mathrm{U}}\right\}$.

ТЕОРема 2.6. 1) Всякая бинарная последовательность с бесконечным числом единии является характеристической для некоторого сильно бескубного $Z$-слова конечного порядка.

2) Два сильно бескубных Z-слова конечного порядка изоморфны тогда и только тогда, когда их характеристические последовательности почти совпадают (т.е. различаются в конечном числе точек).

СлЕДСТВИЕ 2.7 [5]. Язык $O F^{Z}$ содержит континуум попарно неизоморфных $Z$-слов.

ДокАЗАтЕльство. Рассмотрим множество всех бинарных последовательностей (их континуум) и отношение эквивалентности на нем: две последовательности эквивалентны тогда и только тогда, когда сушествует номер $N$, начиная с которого они совпадают. Последовательности можно рассматривать как $\omega$-слова; каждое $\omega$-слово, совпадающее с данным с $N$-й буквы, однозначно определяется своим префиксом длины $(N-1)$; таким образом, класс эквивалентности данного слова инъективно отображается в $A^{+}$, т.е. счетен. Следовательно, классов эквивалентности континуум. Согласно п. 2) теоремы 2.6 требуемое утверждение доказано. 
2.4. Доказательство теоремы 2.6, 2). Нам будет удобнее доказать вначале п. 2). Пусть $\mathbf{U} \neq \mathbf{V} \in O F^{Z}$. Доказательство естественным образом разбивается на несколько этапов. Сначала докажем необходимость.

Этап 1. Если $\mathbf{V}=\operatorname{Aut}(\mathbf{U})$, то порядки всех точек относительно $\mathbf{U}$ и $\mathbf{V}$ совпадают, т.е. совпадают и характеристические последовательности этих $Z$-слов.

Этап 2. Покажем, что если $\mathbf{V}=\operatorname{Shift}_{m}(\mathbf{U})$, то существует номер $N$, начиная с которого последовательности $\left\{h_{n}^{\mathbf{U}}\right\}$ и $\left\{h_{n}^{\mathbf{V}}\right\}$ совпадают. Пусть $k=\max \left\{\operatorname{deg}_{\mathbf{U}}(x) \mid\right.$ $\left.x \in\left[x_{0}, x_{0}+m\right]\right\}$. Рассмотрим точку $x_{1}$ порядка $k$ из отрезка $\left[x_{0}, x_{0}+m\right]$, а также точки $x_{2}, x_{3}-$ ближайшие к $x_{1}$ точки порядка $\geqslant k$. Согласно следствию 2.3 одна из них (пусть это $x_{2}$ ) имеет порядок $(k+1)$, а другая - порядок $\geqslant(k+2)$. В итоге картина такова (с точностью до переименования $x_{2}$ и $x_{3}$ ):

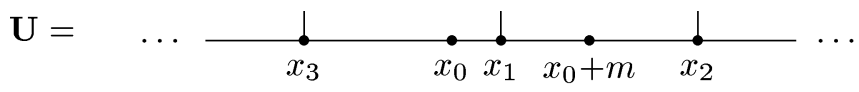

Видно, что $x_{3}$ является ближайшей точкой порядка $\geqslant k+2$ как к точке $x_{0}$, так и к точке $x_{0}+m$. Пусть порядок $x_{3}$ равен $j$. Тогда ближайшей к $x_{0}$ и $x_{0}+m$ точкой порядка $(j+1)$ будет точка $x_{3}+2^{j}$. Рассуждая по индукции, получаем, что $y_{n}^{\mathrm{U}}=y_{n}^{\mathrm{V}}+m$ при всех $n \geqslant k+2$. Следовательно,

$$
\left|y_{n+1}^{\mathrm{U}}-y_{n}^{\mathrm{U}}\right|=\left|y_{n+1}^{\mathrm{V}}-y_{n}^{\mathrm{V}}\right|,
$$

откуда $h_{n}^{\mathbf{U}}=h_{n}^{\mathbf{V}}$. Таким образом, можно взять $N=k+2$.

Этап 3. На втором этапе мы фактически показали, что характеристическая последовательность $Z$-слова и аналогичная последовательность, построенная исходя из произвольной точки $x_{0}^{\prime}$, совпадают, начиная с некоторого элемента (при этом нам не было важно, что $\left|x_{0}-x_{0}^{\prime}\right|$ - целое число). Рассмотрим $Z$-слово $\operatorname{Rev}(\mathbf{U})$. Очевидно, что, взяв за исходную для построения точку $x_{0}^{\prime}=-1 / 3$, мы получим последовательность, совпадающую с $\left\{h_{n}^{\mathrm{U}}\right\}$. Следовательно, характеристические последовательности $Z$-слов $\mathbf{U}$ и $\operatorname{Rev}(\mathbf{U})$ совпадают, начиная с некоторого номеpa $N$. Отметим, что для всех $n \geqslant N$ выражения $y_{n}^{\mathbf{U}}-x_{0}$ и $y_{n}^{\operatorname{Rev}(\mathbf{U})}-x_{0}$ имеют противоположные знаки.

Итак, мы проверили требуемые условия для всех элементарных операций - доказательство необходимости закончено. Докажем достаточность.

Этап 4. Предположим, что $\left\{h_{n}^{\mathbf{U}}\right\}=\left\{h_{n}^{\mathbf{V}}\right\}$, и покажем, что $\mathbf{V}=\operatorname{Aut}(\mathbf{U})$.

В самом деле, из равенства характеристических последовательностей следует, что порядки всех точек относительно $\mathbf{U}$ и $\mathbf{V}$ также равны. Если условие $\mathbf{V}=$ $\operatorname{Aut}(\mathbf{U})$ на выполняется, то найдется такая точка $x$, что в $\mathbf{U}$ и $\mathbf{V}$ либо совпадают буквы с номером $x$ и не совпадают буквы с номером $(x+1)$, либо наоборот. Без ограничения обшности можно считать, что имеет место следуюшая картина:

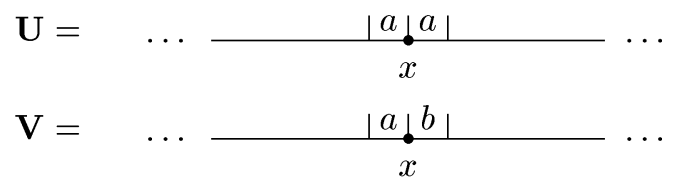


По определению $\operatorname{deg}_{\mathbf{U}}(x-1)=0$, откуда $\operatorname{deg}_{\mathbf{U}}(x) \geqslant 1$ по следствию 2.3. Это означает, что картина такова:

$$
\begin{aligned}
& \mathbf{U}=\quad \cdots \frac{\mid b_{\mid} a_{\downarrow} a_{\mid} b_{\mid}}{x} \cdots \\
& \mathbf{V}=\quad \cdots \frac{\left|b_{\mid} a_{b} b\right| a \mid}{x} \cdots
\end{aligned}
$$

Теперь по определению получаем $\operatorname{deg}_{\mathbf{V}}(x-2)=1$, т.е. $\operatorname{deg}_{\mathbf{V}}(x) \geqslant 2$ по следствию 2.3. Рассуждая по индукции, мы получим, что для любого $k$ порядок точки $\left(x-2^{k}\right)$ равен $k$; отсюда по следствию 2.3 порядок $x$ строго больше $k$. Таким образом, $x$ - точка порядка $\infty$; противоречие с условием.

Этап 5. Пусть теперь у $Z$-слов $\mathbf{U}$ и $\mathbf{V}$ совпадают характеристические последовательности, начиная с $k$-го члена. Рассмотрим вначале случай, когда знаки разностей $y_{k}^{\mathrm{U}}-x_{0}$ и $y_{k}^{\mathrm{V}}-x_{0}$ совпадают:

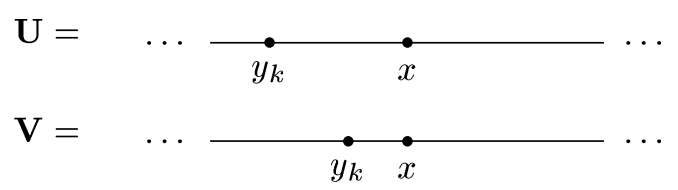

Так как $h_{n}^{\mathbf{U}}=h_{n}^{\mathbf{V}}$ при любом $n \geqslant k$, то разности $y_{n}^{\mathbf{U}}-x_{0}$ и $y_{n}^{\mathbf{V}}-x_{0}$ также будут одного знака; следовательно, равенство

$$
\left|y_{n+1}^{\mathrm{U}}-y_{n}^{\mathrm{U}}\right|=\left|y_{n+1}^{\mathrm{V}}-y_{n}^{\mathrm{V}}\right|
$$

остается верным и при снятии модулей. Пусть $m=y_{k}^{\mathrm{U}}-y_{k}^{\mathrm{V}}$. Для всех точек $x$ таких, что $\operatorname{deg}_{\mathbf{U}}(x) \geqslant k$, выполняется

$$
\operatorname{deg}_{\mathbf{U}}(x)=\operatorname{deg}_{\operatorname{Shift}_{m}(\mathbf{V})}(x) .
$$

Согласно следствиям 2.2 и 2.3 порядок $k-1$ относительно двух данных слов имеют в точности все точки вида $x+2^{k-1}$, где $x$ - точка порядка $\geqslant k$. Таким образом, по индукции получаем, что порядки всех точек относительно $\mathbf{U}$ и Shift $_{m}(\mathbf{V})$ совпадают. Согласно доказанному на четвертом этапе

$$
\mathbf{U} \in\left\{\operatorname{Shift}_{m}(\mathbf{V}), \operatorname{Aut}\left(\operatorname{Shift}_{m}(\mathbf{V})\right)\right\} .
$$

Этап 6. Осталось рассмотреть случай, когда знаки разностей $y_{k}^{\mathrm{U}}-x_{0}$ и $y_{k}^{\mathrm{V}}-x_{0}$ противоположны:

$$
\begin{aligned}
& \mathbf{U}=\cdots \frac{\dot{y}_{k}}{x^{*}} \\
& \mathbf{V}=\cdots
\end{aligned}
$$

Как отмечалось выше (этап 3 ), характеристические последовательности $Z$-слов $\mathbf{V}$ и $\operatorname{Rev}(\mathbf{V})$ совпадают с некоторого номера $N$, причем для всех $n>N$ выражения $y_{n}^{\mathbf{V}}-x_{0}$ и $y_{n}^{\operatorname{Rev}(\mathbf{V})}-x_{0}$ имеют противоположные знаки. Таким образом, согласно доказанному на предыдущем этапе $Z$-слово $U$ совпадает либо с подходящим сдвигом $\operatorname{Rev}(\mathbf{V})$, либо с автоморфизмом такого сдвига.

Доказательство п. 2) теоремы завершено. 
2.5. Доказательство теоремы $2.6,1)$. Определим методпостроения $Z$-слов, которьй мы называем обобщенным методом раскачивания.

Пусть $d \in\{0,1\}$, а $\left\{\gamma_{i}\right\}_{1}^{\infty}$ - произвольная последовательность натуральных чисел. Положим $x_{0}=(2-d) / 3, W_{0}=\mathbf{W}_{[1]}=a$ и определим индуктивно $i$-й шаг. Пусть слово $W_{i-1}$ являлось $m$-цепочкой. На $i$-м шаге к этому слову приписывается (с той стороны, которая ближе к точке $\left.x_{0}\right) 2^{\gamma_{i}}-1$ штук $m$-цепочек, причем так, чтобы полученное слово $W_{i}$ являлось $\left(m+\gamma_{i}\right)$-цепочкой (это можно осуществить единственным способом).

Заметим, что всякое $W_{k}$ является в точности $\Gamma_{k}$-цепочкой, где $\Gamma_{k}=\sum_{i=1}^{k} \gamma_{i}$.

Какую бы последовательность $\left\{\gamma_{i}\right\}$ мы не выбрали, данный метод приводит к построению $Z$-слова из $O F^{Z}$ (всякое подслово полученного $Z$-слова лежит в некотором $\left.W_{k} \in T M\right)$; при этом последовательность, состоящая из единищ, приводит к построению $Z$-слова по обычному методу раскачивания.

Пусть $\left\{h_{n}\right\}_{0}^{\infty}-$ бинарная последовательность с бесконечным числом единиц. Выберем $d$ и $\left\{\gamma_{n}\right\}_{1}^{\infty}$ для построения $Z$-слова обобщенным методом раскачивания. Пусть $d=h_{0}, \gamma_{1}$ есть номер первой единицы в $\left\{h_{n}\right\}_{1}^{\infty}$, а для $k>1$ число $\gamma_{k}$ есть разность номеров $k$-й и $(k-1)$-й единиц в $\left\{h_{n}\right\}_{1}^{\infty}$ (т.е. в $\left\{h_{n}\right\}_{1}^{\infty}$ единицы стоят в точности на всех позициях и с номерами, равными частичным суммам последовательности $\left.\left\{\gamma_{n}\right\}\right)$.

Пусть обобщенный метод строит по $d$ и $\left\{\gamma_{n}\right\} \quad Z$-слово $\mathbf{W}$. Проверим, что последовательность $\left\{h_{n}\right\}$ характеристическая для $\mathbf{W}$.

Точку $x$ назовем $k$-крайней, если при построении $\mathbf{W}$ она являлась конщом отрезка, занимаемого $W_{k}$, но попадала внутрь отрезка, занимаемого $W_{k+1}$. Покажем, что $k$-крайняя точка имеет порядок $\Gamma_{k}$. В самом деле, слово $W_{k}$ является $\Gamma_{k}$-цепочкой; при дальнейшем построении добавляются блоки, являюшиеся произведением, по меньшей мере, $\Gamma_{k}$-цепочек. Таким образом, порядок $k$-крайней точки не меньше $\Gamma_{k}$ по определению. Далее, легко проверить, что другим концом отрезка, занимаемого $W_{k}$, является $(k+1)$-крайняя точка. Ее порядок строго больше $\Gamma_{k}$, так как $\gamma_{k+1}>0$. Но расстояние между концами этого отрезка равно $2^{\Gamma_{k}}$, откуда по следствию 2.3 порядок $k$-крайней точки в точности равен $\Gamma_{k}$.

Теперь построим характеристическую последовательность для $\mathbf{W}$. Заметим, что $k$-крайняя точка $\left(k \geqslant 1\right.$ ) является ближайшей к $x_{0}=1 / 3$ (и вообще к любой точке интервала $(0,1))$ точкой порядка $\geqslant \Gamma_{k}$. Более того, ближайшая к $x_{0}$ точка порядка $\geqslant \Gamma_{k}+1(k \geqslant 0)$ отстоит от $k$-крайней точки на расстоянии $2^{\Gamma_{k}}$ и, следовательно, совпадает с $(k+1)$-крайней точкой:

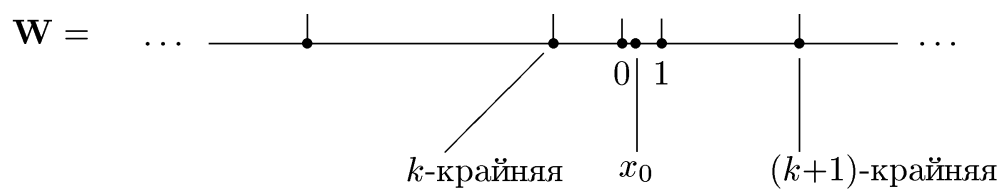

Таким образом, $y_{1}, \ldots, y_{\Gamma_{1}}$ совпадают с 1-крайней точкой, и в общем случае $y_{\Gamma_{k}+1}, \ldots, y_{\Gamma_{k+1}}$ совпадают с $(k+1)$-крайней точкой. Точка $y_{0}$ совпадает, как легко убедиться, с 0 -крайней точкой при $d=1$ и с 1 -крайней в противном случае. Отсюда получаем, что $h_{0}^{\mathrm{W}}=1$ тогда и только тогда, когда $y_{0}-0$-крайняя точка, т.е. $d=1$. Пусть $i>0$. Имеем $h_{i}^{\mathbf{W}}=1$ тогда и только тогда, когда $i=\Gamma_{k}$ для некоторого $k$. В итоге $\left\{h_{n}^{\mathbf{W}}\right\}=\left\{h_{n}\right\}$, что и требовалось доказать. 
Итак, мы завершили доказательство п. 1), а с ним и всей теоремы.

ЗАмЕчАниЕ. Из доказательства п. 1) следует, что всякое сильно бескубное $Z$-слово конечного порядка (с точностью до автоморфизма) можно построить обобщенным методом раскачивания по подходящей бинарной последовательности с бесконечным числом единиц.

2.6. Заключительные замечания. Бинарные последовательности из "почти нулевого" класса (т.е. с конечным числом единиц) являются характеристическими для $Z$-слов бесконечного порядка. Таким образом, этому "вырожденному" классу можно поставить в соответствие два вырожденных класса $Z$-слов бесконечного порядка. Этим завершена характеризация сильно бескубных $Z$-слов на языке бинарных последовательностей.

Если теперь заметить, что бинарные последовательности суть то же самое, что $\omega$-слова в двухбуквенном алфавите, то мы получим впечатляюшую разницу в масштабах между множествами $Z$-слов и $\omega$-слов:

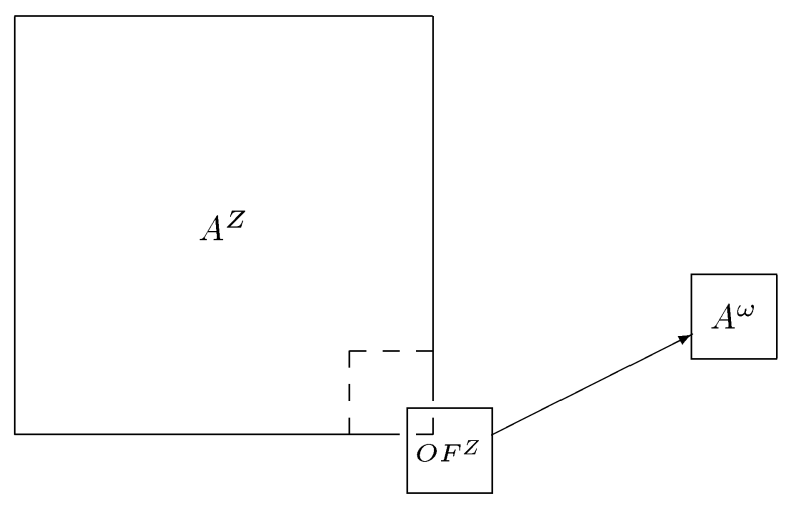

\section{§3. Языки, промежуточные между $O F^{Z}$ и $C F^{Z}$}

В предыдущем параграфе мы описали структуру языка, являющегося самой нижней ступенью иерархии, введенной в п. 1.3. Основная цель данного параграфа - установить, какие из языков вида $L_{\beta}, L_{\beta}^{\prime}$ различны, а какие совпадают. Таким образом мы выясним, сколько различных ступеней в данной иерархии и как они расположены между $O F^{Z}$ и $C F^{Z}$. Мы также докажем одно свойство языка, являющегося второй ступенью иерархии, демонстрирующее глубокое различие между данным языком и языком $O F^{Z}$.

\section{1. Язык $L_{\alpha}^{\prime Z}$ - вторая ступень иерархии.}

ПРЕДЛОЖЕНИЕ 3.1. Пусть $\alpha=7 / 3$. Тогда $L_{\alpha}^{Z}=O F^{Z} \subset L_{\alpha}^{\prime Z}$.

ДокАЗАТЕльСтво. Покажем, что все элементы $L_{\alpha}^{Z}$ равномерны. Предположим противное: сушествует неравномерное слово $\mathbf{W} \in L_{\alpha}^{Z}$. Тогда оно содержит неравномерное подслово $U$. Легко проверить, что конечное слово неравномерно тогда и только тогда, когда оно содержит подслова $c c$ и $d d, c, d \in A$, одно из которых начинается в $U$ с нечетной позиции, а другое - с четной. Рассмотрим $V \leqslant U$ такое, что $V$ имеет префикс $c c$, суффикс $d d$ и не содержит других подслов $c c, d d$. 
Тогда $V$ либо совпадает с одним из слов $a a a / b b b / a a b a a / b b a b b$, либо содержит подслово $a b a b a(b a b a b)$. В итоге $\mathbf{W}$ необходимо содержит хотя бы одно из слов

$$
a a a, b b b, \text { abbabba, baabaab, ababa, babab, }
$$

каждое из которых имеет экспоненту, не меньшую $7 / 3$. Противоречие с условием $\mathbf{W} \in L_{\alpha}^{Z}$.

Таким образом, все элементы $L_{\alpha}^{Z}$ допускают разбиение на 1-цепочки (ср. предложение 2.1). По предложению $1.2 Z$-слово, получаемое применением к элементу $L_{\alpha}^{Z}$ операции $\phi^{-1}$, также принадлежит $L_{\alpha}^{Z}$. Рассуждая по индукции, как в предложении 2.1 , получаем, что всякое $\mathbf{W} \in L_{\alpha}^{Z}$ допускает разбиение на $n$-цепочки для всех натуральных $n$. Таким образом, всякое подслово из $\mathbf{W}$ принадлежит $T M$, т.е. $L_{\alpha}^{Z}=O F^{Z}$.

В отличие от элементов $L_{\alpha}^{Z}$, элементы $L_{\alpha}^{\prime Z}$ могут содержать подслова $a b b a b b a$, baabaab. Примером элемента множества $L_{\alpha}^{\prime Z} \backslash O F^{Z}$ может служить $Z$-слово ${ }_{\infty} U a b a U_{\infty}$

Таким образом, в исследуемой иерархии есть вторая ступень - язык $L_{\alpha}^{\prime Z}$. Число $\alpha=7 / 3$ зафиксируем до конца параграфа, чтобы уменьшить громоздкость некоторых обозначений.

Сейчас укажем одно принщипиальное различие между языком $L_{\alpha}^{\prime Z}$ и языком $O F^{Z}$, занимающим нижнюю ступень иерархии.

Слово над алфавитом $A$ называется уравновешенным, если $|W|_{a}=|W|_{b}$ (через $|W|_{c}$, где $c \in A$, обозначается число букв $c$ в слове $\left.W\right)$. $Z$-слово $\mathbf{W}$ над $A$ назовем уравновешенным уровня $n$, если для всякого (конечного) его подслова $W$ выполняется неравенство $\left.|| W\right|_{a}-|W|_{b} \mid \leqslant n$.

Легко заметить, что все равномерные $Z$-слова и, в частности, все элементы $O F^{Z}$ являются уравновешенными уровня 2 . В то же время для языка $L_{\alpha}^{\prime Z}$ картина совсем иная, как показывает следуюшая теорема.

Теорема 3.2. В языке $L_{\alpha}^{\prime Z}$ есть неуравновешеннье $Z$-слова.

3.2. Доказательство теоремы 3.2. Укажем метод построения требуемого $Z$-слова. Легко заметить, что слово baabaab, имеющее экспоненту $7 / 3$, может встречаться в $Z$-словах из $L_{\alpha}^{\prime Z}$ только внутри сегмента $W=a b b a a b a a b b a$. Имеем $|W|_{a}-|W|_{b}=1$. Построим $Z$-слово $\mathbf{W}$, соединив счетный набор сегментов $W$ специальным образом подобранными уравновешенными словами-связками. Показав затем, что $\mathbf{W} \in L_{\alpha}^{\prime Z}$, мы докажем данную теорему.

Возьмем в качестве связок слова

$$
\begin{aligned}
& X_{1}=V_{4} \quad=b a a b a b b a \quad a b b a b a a b, \\
& X_{2}=V_{3} V_{2} V_{2}=b a a b \quad a b b a \text { baab baab, } \\
& X_{3}=V_{2} V_{2} V_{3}=b a a b \text { baab abba baab, }
\end{aligned}
$$

и пусть $\Delta$ - произвольное бесквадратное $Z$-слово над трехбуквенным алфавитом $\{1,2,3\}$. Теперь составим $Z$-слово из блоков $X_{i}$, полагая $k$-й блок равным $X_{\Delta[k]}$. Между каждой парой соседних блоков полученного $Z$-слова вставим слово $W$. Итоговое $Z$-слово и будет искомым $\mathbf{W}$. Говоря в дальнейшем о разбиении $\mathbf{W}$, мы будем иметь в виду разбиение на блоки $X_{i}$ и $W$, индуцированное построением. Сразу отметим, что $X_{i} \in T M$, откуда $\operatorname{lexp}\left(X_{i}\right)=2$ и $X_{i}$ - равномерное слово. Отметим также, что $\operatorname{lexp}(W)=7 / 3,|W|=11,\left|X_{i}\right|=16$. 
Докажем, что $\mathbf{W}=L_{\alpha}^{\prime Z}$. Покажем, что всякое подслово $P \leqslant \mathbf{W}$ имеем экспоненту, не превышаюшую $7 / 3$. Предположим, что $P=X Y X Y X($ т.е. $\exp (P)>2)$ и $P$ пересекается с блоками $T^{(1)}, \ldots, T^{(k)}$ разбиения $\mathbf{W}$ :

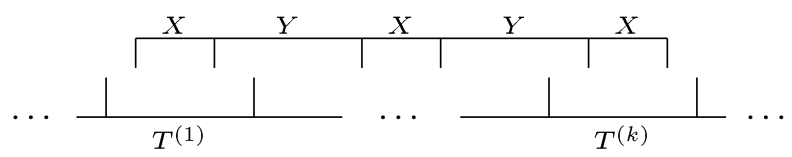

При малых $k$ выполнение требуемого условия будет обеспечиваться за счет устройства слов $W$ и $X_{i}$, а при больших $k \geqslant 6$ - за счет бесквадратности $\Delta$.

Доказательство разделим на семь этапов.

Этап 1 . При $k \leqslant 2$ требуемое условие на $\exp (P)$ вьполняется.

a) $k=1$. Так как $\operatorname{lexp}\left(X_{i}\right)=2, \operatorname{lexp}(W)=7 / 3$, то $\exp (P) \leqslant 7 / 3$.

б) $k=2$. Покажем, что $\operatorname{lexp}\left(W X_{i}\right) \leqslant 7 / 3$. Неравенство $\operatorname{lexp}\left(X_{i} W\right) \leqslant 7 / 3$ проверяется точно так же.

Пусть $P \leqslant W X_{i}, \exp (P)>2$. Предположим вначале, что aаbaa $\leqslant P$. Так как $W X_{i}$ содержит ровно одно подслово аabaа $\left(X_{i}\right.$ - равномерное слово), то $\operatorname{per}(P)<$ $|a a b a a|=5$. Тогда, очевидно, $\operatorname{per}(P)=3$, откуда $P \leqslant W, P=b a a b a a b, \exp (P)=$ $7 / 3$. Если же $P$ не содержит аabaa, то $P \leqslant a b a a b b a X_{i}$. Рассмотрим слова $Y_{i}=$ baabbaX : $^{2}$

$$
Y_{i} \leqslant a b b a a b b a X_{i}=\phi^{2}\left(a a \phi^{-2}\left(X_{i}\right)\right) .
$$

Таким образом, $Y_{1}=\phi^{2}(a a b a a b), Y_{2}=\phi^{2}(a a b a b b), Y_{3}=\phi^{2}(a a b b a b)$. Так как все слова в скобках имеют локальную экспоненту 2 , то $\operatorname{lexp}\left(Y_{i}\right)=2$ по предложению 1.2. Теперь видно, что число $\operatorname{lexp}\left(a Y_{i}\right)$ меньше $7 / 3$, что и требовалось доказать.

Этап 2. Требуемое условие на $\exp (P)$ вьполняется при $k=3$.

Рассмотрим сначала слово вида $X_{i} W X_{j}$. Если в нем есть подслово $P$ экспоненты, большей $7 / 3$, то согласно результатам этапа $1 W \leqslant P$. Тогда аabaa $\leqslant P$ и $\operatorname{per}(P) \geqslant 5$. Следовательно, $P$ содержит еще одно подслово $a a b a a$; однако в слове $X_{i} W X_{j}$ такое подслово всего одно. Данное противоречие показывает, что $\exp (P) \leqslant 7 / 3$.

Теперь рассмотрим слово вида $W X_{i} W$. Аналогично, если оно содержит подслово $P$ экспоненты, большей $7 / 3$, то $X_{i} \leqslant P$. Если $i \neq 1$, то baabbaab $\leqslant P$. Так как $\operatorname{per}(P) \geqslant \operatorname{per}\left(X_{i}\right)=12$, то $P$ должно содержать еще одно подслово $b a a b b a a b$; получаем противоречие, так как таких подслов в $W X_{i} W$ больше нет. Случай $i=1$ рассмотрен на рисунке. Здесь $P$ - наибольшее подслово в $W X_{i} W$ с периодом 12 ; его экспонента в точности равна $7 / 3$ :

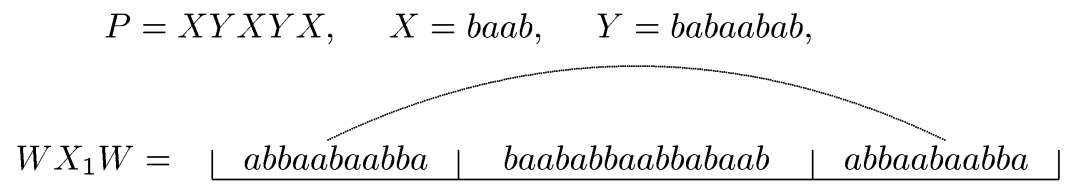

Итак, мы доказали, что $\exp (P) \leqslant 7 / 3$ при $k \leqslant 3$. Для того чтобы доказать требуемое для $k=4, k=5$, нужно вычислить периоды некоторых подслов из $\mathbf{W}$. Этому посвящен следующий этап. 
Этап 3. а) $\operatorname{per}\left(X_{i} W\right)>21$. В самом деле, слово $X_{i} W$ содержит ровно одно подслово aabaa (см. рисунок ниже). Таким образом, это подслово не может входить в суффикс слова $X_{i} W$ длины $\left(\left|X_{i} W\right|-\operatorname{per}\left(X_{i} W\right)\right)$ (в противном случае aаbаa входит в $X_{i} W$ еще раз, на $\operatorname{per}\left(X_{i} W\right)$ букв левее). Следовательно, $\operatorname{per}\left(X_{i} W\right)>19$. Далее замечаем, что если $X_{i}$ имеет префикс baabba $(i=3)$, то $\operatorname{per}\left(X_{i} W\right)=21$, а если префикс baabab, то $\operatorname{per}\left(X_{i} W\right)=25$.

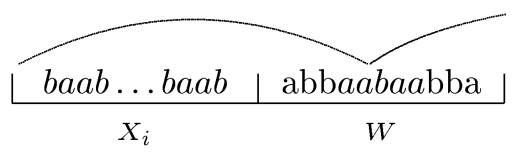

Симметричное рассуждение показывает, что $\operatorname{per}\left(W X_{i}\right) \geqslant 21$.

б) $\operatorname{per}\left(b a X_{i} W\right)=27$. Аналогично п. а) единственность подслова aаbaa в рассматриваемом слове влечет неравенство $\operatorname{per}\left(b a X_{i} W\right)>21$. Заметив, что слово $b a b a$ является префиксом рассматриваемого слова и не встречается справа от aаbaa, мы получаем требуемое:

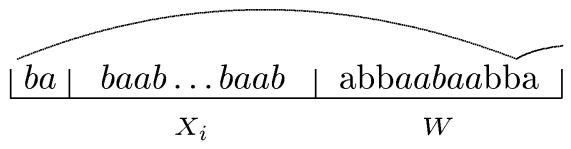

Симметрично получаем $\operatorname{per}\left(W X_{i} a b\right)=27$.

в) $\operatorname{per}\left(W X_{i} W\right)=27$. В самом деле, $27=\left|W X_{i}\right|$, т.е. слово $W X_{i} W$ имеет такой период; с другой стороны, $b a X_{i} W \leqslant W X_{i} W$ и согласно п. б) $\operatorname{per}\left(W X_{i} W\right) \geqslant 27$.

г) Слово $W X_{i} W$ не имеет периодов, меньших 34 , за исключением минимального (непосредственно проверяется).

д) $\operatorname{per}\left(X_{i} W X_{j}\right)>27$. Пользуясь а), получаем, что $\operatorname{per}\left(X_{i} W X_{j}\right) \geqslant 21$. Далее, непосредственно устанавливается, что $\operatorname{per}\left(X_{i} W b\right)=27$, откуда следует $\operatorname{per}\left(X_{i} W X_{j}\right) \geqslant 27$. Но в случае $X_{i} W X_{j} \leqslant \mathbf{W}$ выполняется $i \neq j$ (в силу бесквадратности $\Delta)$. В итоге $\operatorname{per}\left(X_{i} W X_{j}\right)>\left|X_{i} W\right|=27$.

Этап 4. Ограничение на $\exp (P)$ выполняется при $k=4,5$. Пусть $k=4$. Тогда $|P| \leqslant 2|W|+2\left|X_{i}\right|=54$. Но сегмент $T^{(2)} T^{(3)}$, состоящий из слова $W$ и связки, не имеет периодов, меньших 21 (этап 3 ). Таким образом, экспонента $P$ может превосходить $7 / 3$ только при $|P|>49$. Но в этом случае $P$ обязательно содержит сегмент $b a X_{i} W$ или $W X_{i} a b$, каждый из которых имеет период не менее 27 (этап 3$)$. В итоге $\exp (P) \leqslant 7 / 3$.

Пусть $k=5$. Если $T^{(1)}=W$, то $\operatorname{per}(P) \geqslant \operatorname{per}\left(X_{i} W X_{j}\right)>27$ (этап 3$)$, а $|P| \leqslant 3|W|+2\left|X_{i}\right|=65$. Ограничение на экспоненту $P$ выполняется.

Если же $T^{(1)}$ является связкой, то период $P$ является периодом слова $W X_{i} W$. По результатам этапа $3 \operatorname{per}\left(W X_{i} W\right)=27$, а следуюший по величине период этого слова не меньше 34 . Имеем $|P| \leqslant 3\left|X_{i}\right|+2|W|=70$. Так как 70/34 $\leqslant 7 / 3$, то достаточно проверить случай $\operatorname{per}(P)=27$. Две разные связки имеют равные префиксы длины не более 8 и равные суффиксы также длины $\leqslant 8 ;$ следовательно, подслово в $X_{i} W X_{j} W X_{m}$ с периодом 27 не может иметь длину, большую, чем

$$
8+|W|+\left|X_{j}\right|+|W|+8=54 .
$$


Ниже приведен фрагмент слова $X_{3} W X_{1} W X_{2}$, на котором это значение достигается:

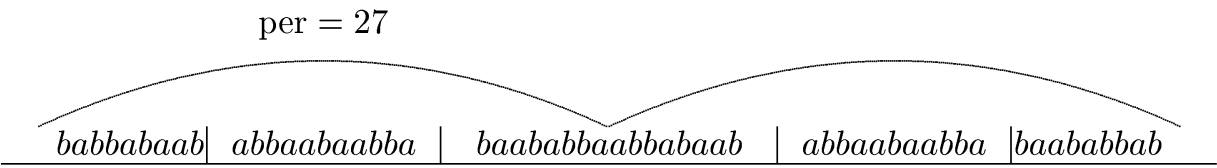

Итак, мы доказали требуемое и для $k=5$.

Теперь докажем утверждение, позволяющее получить желаемую оценку при $k \geqslant 6$.

Этап 5. Пусть $U \leqslant \mathbf{W}, U$ имеет собственный префикс $X_{i_{1}} W X_{i_{2}} W$. Тогда $\exp (U)<2$.

Экспонента слова $X_{i_{1}} W X_{i_{2}} W$ меньше двух, так как согласно этапу 3 $\operatorname{per}\left(X_{i_{1}} W X_{i_{2}}\right)>\left|X_{i_{1}} W\right|$. Предположим, что $U=X Y X Y X$ (возможно, $X=\lambda$ ). Тогда $X_{i_{1}} W$ есть префикс $X Y$, так как $\operatorname{per}\left(X_{i_{1}} W X_{i_{2}}\right)>\left|X_{i_{1}} W\right|$. Второе вхождение $X Y$ в $U$ также начинается с $X_{i_{1}} W$; рассмотрим, как оно размешается в $\mathbf{W}$. Так как $\mathbf{W}$ содержит по одному подслову $a a b a a$ внутри каждого блока разбиения, равного $W$, и не содержит других подслов $a a b a a$, то сегмент $W$ во втором вхождении $X_{i_{1}} W$ должен совпадать с блоком разбиения. Следовательно, и $X_{i_{1}}$ совпадает с некоторой связкой $X_{i_{k+1}}$. Таким образом, $X Y=X_{i_{1}} W \ldots X_{i_{k}} W$. Тогда $i_{1}=i_{k+1}, \ldots, i_{k}=i_{2 k}$. Получаем противоречие с бесквадратностью $Z$-слова $\Delta$. Итак, $\exp (U)<2$.

Этап 6. Аналогично доказанному на этапе $5, \exp (U)<2$ для всякого слова $U \leqslant \mathbf{W}$, имеющего префикс $W X_{i_{1}} W X_{i_{2}}$. Далее, симметричные рассуждения приводят к той же оценке для экспоненты всякого подслова из $\mathbf{W}$, имеющего суффикс, равный одному из приведенных префиксов.

Этап 7. Пусть теперь $k \geqslant 6$. Воспользовавшись результатами этапов 5,6 , получаем, что префикс слова $P=X Y X Y X$, заканчивающийся блоком $T^{(k-1)}$, и суффикс этого слова, начинаюшийся блоком $T^{(2)}$, имеют экспоненту, меньшую двух. Следовательно, слово $X Y X Y$ пересекается с $T^{(k)}$, а слово $Y X Y X-$ с $T^{(1)}$. Таким образом, $X$ является собственным подсловом как в $T^{(1)}$, так и в $T^{(k)}$. Отсюда следует, что $|X| \leqslant 14$. Заметим также, что $\operatorname{per}(P)>\left|T^{(2)} \ldots T^{(k-1)}\right| / 2 \geqslant\left|X_{i}\right|+$ $|W|=27$. Таким образом, при $|X| \leqslant 9$ все уже доказано.

Пусть $|X| \geqslant 10$. Тогда $T^{(1)}$ и $T^{(k)}$ - связки, т.е. $k$ должно быть нечетным. Осталось заметить, что при таком условии $P$ содержит подслово $W X_{i} W X_{j} W(i \neq$ $j)$, минимальньй период которого равен $\left|W X_{i} W X_{j}\right|$, т.е. 54 . Так как $|X| \leqslant 14$, то и в этом случае экспонента $P$ не превосходит $7 / 3$. Теорема доказана.

Заметим, что можно построить континуум различных неуравновешенных $Z$ слов, принадлежаших множеству $L_{\alpha}^{\prime Z}$, так как существует континуум различных бесквадратных $Z$-слов в трехбуквенном алфавите (см., например, [11]).

\section{3. Структура иерархии выше языка $L_{\alpha}^{\prime Z}$.}

Теорема 3.3. Пусть $\beta$ - рациональное число, $7 / 3<\beta<3$. Тогда существует $Z$-слово $\mathbf{W}$ такое, что $\operatorname{lexp}(\mathbf{W})=\beta$. 
СлЕДСТВИЕ 3.4. Все языки $L_{\gamma}^{Z}$, где $\gamma \in[7 / 3,3]$, различны.

ДокАЗАТЕЛЬСТво. Пусть $\gamma_{1}<\gamma_{2}$; тогда сушествует рациональное $\beta$ такое, что $\gamma_{1}<\beta<\gamma_{2}$. Слово экспоненты $\beta$, существуюшее по теореме 3.3 , принадлежит $L_{\gamma_{2}}^{Z} \backslash L_{\gamma_{1}}^{Z}$.

Таким образом, данная теорема и следствие проясняют вопрос о мощности исследуемой иерархии языков (она континуальна) и демонстрируют, что ступени иерархии расположены всюду плотно для локальных экспонент из отрезка $[7 / 3,3]$. Вопрос о строгости включения $L_{\gamma}^{Z} \subseteq L_{\gamma}^{\prime Z}$ для иррациональных $\gamma$ будет разобран в п. 3.6 .

3.4. Комбинаторные леммы. Нам потребуются три технические леммы.

Лемма 3.5. Пусть $m$ - неотрицательное четное число. Тогда существует слово $R \in T M$ такое, что $|R|=m$ и слово $P=$ aаbRbаа также принадлежит $T M$.

ДокАЗАТЕЛЬСТво. Вначале разберем случай, когда $m$ делится на 4. Заметим, что $P \leqslant V_{2} R V_{2}$. Возьмем $Z$-слова $\infty U V_{\infty}, \infty V V_{\infty}$ и их 2-разбиения (единственные по предложению 2.1). В каждом из этих $Z$-слов рассмотрим блок 2-разбиения, являюшийся префиксом $V_{\infty}$ и равный $V_{2}$. Так как $m$ кратно 4 , то на $m+4$ символа левее этой 2-цепочки в каждом из указанных $Z$-слов также расположена 2 -цепочка; эта последняя цепочка, очевидно, равна $U_{2}$ в одном из $Z$-слов и $V_{2}$ в другом. В итоге одно из указанных $Z$-слов, являюшихся элементами $O F^{Z}$, содержит подслово вида $V_{2} R V_{2}$, где $|R|=m$, а следовательно, и слово $P$.

Пусть теперь $m$ не делится на 4 . Положим $R=R^{\prime} a b$. Тогда $P \leqslant V_{2} R^{\prime} U_{2} U_{2}$ и $\left|R^{\prime}\right|$ кратно 4 . Возьмем те же $Z$-слова, что и в предыдушем случае, и рассмотрим самый левый сегмент $U_{2} U_{2}$ в $\omega$-слове $V_{\infty}$. Непосредственно слева от него расположена цепочка $V_{2}$, являюшаяся префиксом $V_{\infty}$. Как было показано выше, на любом расстоянии, кратном 4 , слева от этой 2-цепочки, одно из выбранных $Z$-слов содержит 2 -цепочку $V_{2}$. В итоге получаем, что слово $V_{2} R^{\prime} U_{2} U_{2}$, а с ним и слова $R$ и $P$ принадлежат $T M$. Лемма доказана.

Две оставшиеся леммы характеризуют квадраты (слова вида $X X)$, являющиеся подсловами элементов $O F$ и $T M$. Доказательство первой из них приведено в [10], а второй - в [12].

Лемма 3.6. Пусть $X X \in O F . \quad$ Тогдa $|X|=2^{k}$ или $|X|=3 \cdot 2^{k} \partial л я$ подходящего $k$.

Лемма 3.7. Пусть $X X \leqslant U_{n}$. Если $|X|=2^{k}$, то слово $X$ является $k$-цепочкой и позичия $1_{X}$ в $U_{n}$ кратна $2^{k}$. Если $|X|=3 \cdot 2^{k}$, mо $X=U_{k} V_{k} U_{k}$ $\left(X=V_{k} U_{k} V_{k}\right)$ и позичия $1_{X}$ в $U_{n}$ кратна $2^{k}$.

3.5. Доказательство теоремы 3.3. Для произвольного слова $R \in A^{+}$зафиксируем следующее свойство:

$(*)$ у всех подслов $R$, минимальный период которых меньше $\operatorname{per}(R)$, әкспонента не превосходит $7 / 3$.

Пусть, как и в предыдущей теореме, $W=a b b a a b a a b b a$, и пусть $m \geqslant 4$. На первом этапе доказательства построим слово $U$, состоящее из $m 2$-цепочек такое, что слово $T=\left(W U^{3}\right)$ обладает свойством $(*)$. Очевидно, что первая 2-цепочка в $U$ не равна $a b b a$ (в противном случае слово $W U$ содержит подслово baabbaabba экспоненты $5 / 2)$. Аналогично, последняя 2 -цепочка в $U$ также не равна $a b b a$. Таким 
образом, $U=b a a b Z b a a b$, где $Z$ также является произведением 2-цепочек. Чтобы указать подходящее $Z$, рассмотрим слово $V=$ baabbabaabZbaababbaab:

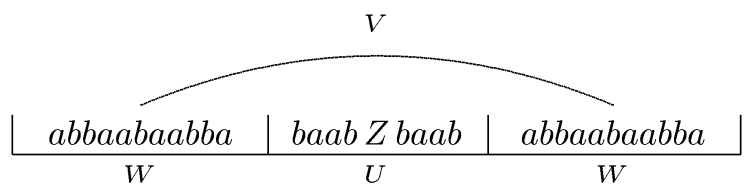

и слово $V^{\prime}=a a b \phi^{-2}(Z) b a a$. Длина слова $Z^{\prime}=\phi^{-2}(Z)$ равна $m-2$ по определению $Z$. Если $m$ - четное число, то выберем $Z^{\prime}$ так, чтобы слово $V^{\prime}$ принадлежало $T M$ (такое $Z^{\prime}$ сушествует по лемме 3.5 ). Если же $m$ нечетно, то $\left|Z^{\prime}\right| \geqslant 3$; положим $Z^{\prime}=a a b Z^{\prime \prime}$.

Снова пользуясь леммой 3.5 , выберем $Z^{\prime \prime}$ так, чтобы $a a b Z^{\prime \prime} b a a \in T M$. Заметим, что $Z^{\prime \prime} \in\{\lambda, a b, b a, a b b a X, b a b a X, b a a b X\}$, где $X$ - некоторое слово из $A^{+} \cup \lambda$. Выясним, чему равна локальная экспонента слова $V^{\prime}=a a b a a b Z^{\prime \prime} b a a$. В случаях $Z^{\prime \prime}=\lambda, Z^{\prime \prime}=b a$ непосредственно вычисляем $\operatorname{lexp}\left(V^{\prime}\right)=2$. Исследуем остальные возможности.

Очевидно, условие $a a b Z^{\prime \prime} b a a \in T M$ влечет $b a a b Z^{\prime \prime} b a a \in T M$. Если слово $Z^{\prime \prime}$ имеет префикс $b a a b$, то $b a a b Z^{\prime \prime} b a a$ не может начинаться квадратом слова длины $>$ 4 согласно лемме 3.7. Значит, слово $a b a a b Z^{\prime \prime} b a a$ не имеет префикса экспоненты, большей 2 , откуда $\operatorname{lexp}\left(a b a a b Z^{\prime \prime} b a a\right)=2$. Далее, заметим, что слово $a b a a b Z^{\prime \prime} b a a$ не начинается квадратом никакого слова. В самом деле, пусть оно имеет префикс $X X$. Непосредственно проверяем, что $|X|>4$. Так как $a b a a b Z^{\prime \prime} b a a \in O F$, из леммы 3.6 получаем, что $|X|$ - четное число. Так как слово $a b a a b Z^{\prime \prime} b a a$ равномерно и имеет разбиение вида $a Q_{1} \ldots Q_{n} d$, то слово $1_{X} 0_{X}$ есть 1-цепочка, т.е. $1_{X}=b$. Но тогда после $X X$ в исследуемом слове снова идет буква $a=0_{X}$. Противоречие с условием $\operatorname{lexp}\left(a b a a b Z^{\prime \prime} b a a\right)=2$. В итоге $\operatorname{lexp}\left(V^{\prime}\right)=2$, так как при отбрасывании первой буквы этого слова мы получаем слово экспоненты 2 , не начинающееся квадратом никакого слова.

Из приведенного рассуждения ясно, что $\operatorname{lexp}\left(V^{\prime}\right)=2$ и при равенстве $Z^{\prime \prime}=$ babaX.

Теперь разберем случай, когда слово $Z^{\prime \prime}$ равно $a b$ или имеет префикс $a b b a$. Тогда слово $b a a b Z^{\prime \prime} b a a \in T M$ не может начинаться квадратом длины, меньшей 8 (напомним, что согласно доказательству леммы $3.5 Z^{\prime \prime}$ есть произведение нескольких 2-цепочек, домноженное справа на цепочку $a b)$. Если это слово имеет префикс $X X,|X|=k \geqslant 8$, то $k$ четное (лемма 3.6 ), $X$ заканчивается 1 -цепочкой, а слово $a b a a b Z^{\prime \prime} b a a$, возможно, имеет префикс экспоненты $2+1 / k$, которая не превосходит $17 / 8$. При этом слово $V^{\prime}$ не может иметь префикс экспоненты $2+2 / k$, так как $X$ заканчивается 1-цепочкой. Далее, слово $V^{\prime}$ состоит из префикса aаbaа и равномерного суффикса; таким образом, оно начинается квадратом длины 3 и не начинается никаким другим квадратом; в итоге $\operatorname{lexp}\left(V^{\prime}\right)=7 / 3$.

Вернемся к словам $U=b a a b Z b a a b$ и $V=b a a b b a U a b b a a b$. Согласно предложению 1.2

$$
\operatorname{lexp}\left(V^{\prime}\right)=\operatorname{lexp}\left(\phi^{2}\left(V^{\prime}\right)\right) \geqslant \operatorname{lexp}(V),
$$

так как $V \leqslant \phi^{2}\left(V^{\prime}\right)$. 
Итак, нами построено слово $U$, состояшее из $m$ 2-цепочек. Покажем, что слово $T=(W U)^{3}$ удовлетворяет свойству $(*)$ :

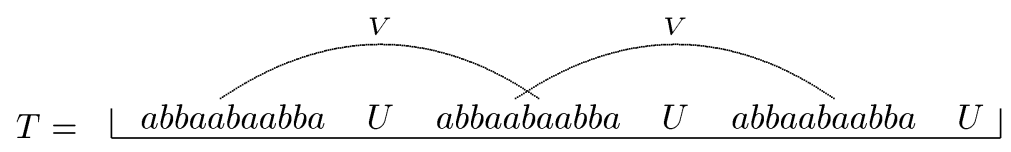

Пусть $P \leqslant T$ и $\exp (P) \geqslant 2$. Если $P$ содержит один сегмент $a a b a a$, то $\operatorname{per}(P)<5$ и, очевидно, $P=b a a b a a b$, т.е. $\exp (P)=7 / 3$. Если $P$ содержит два или три сегмента $a a b a a$, то минимальный период $P$ не меньше расстояния между двумя соседними вхождениями этого сегмента в $T$, а это расстояние равно $\operatorname{per}(T)$. Если же $P$ вообще не содержит сегмента $a a b a a$, то $P \leqslant a V a$, так как этот сегмент не содержится в $U$. Докажем, что и в этом случае $\exp (P) \leqslant 7 / 3$. Это неравенство очевидно выполняется при $\operatorname{lexp}(V)=2$; случай $\operatorname{lexp}(V)>2$ рассмотрим подробнее.

Условие $\operatorname{lexp}(V)>2$ означает, что $\operatorname{lexp}\left(V^{\prime}\right)>2$, что возможно только в случае $V^{\prime}=a a b a a b Z^{\prime \prime} b a a$, когда $0_{Z^{\prime \prime}}=a$. В этом случае $V^{\prime}$ имеет префикс длины $7 \mathrm{c}$ периодом 3 и не имеет других префиксов и суффиксов экспоненты, большей 2 (см. вьше). Тогда само слово $V$ имеет префикс длины $26=7 \cdot 2^{2}-2$ с периодом $12=3 \cdot 2^{2}$ и не имеет других префиксов и суффиксов экспоненты, большей 2. Кроме этого, $V$ не содержит префиксов/суффиксов длины $\leqslant 6$, имеюших экспоненту 2. Этого достаточно, чтобы утверждать, что слово $a V a$ имеет экспоненту не более $7 / 3$.

Итак, свойство (*) выполняется.

Из определения $T$ и свойства $(*)$ следует, что всякий префикс $T$ длины $k \geqslant$ $7|W U| / 3$ имеет локальную экспоненту, равную $k /|W U|$. Попутно сделаем следующее простое наблюдение.

(i) Всякий префикс T, содержащий все три подслова аабаа, не имеет периодов, промежуточных между $|W U|$ и $2|W U|$.

Пусть число $\beta$ представимо в виде несократимой дроби $k /\left(2^{s} \cdot n\right)$, где $n$ нечетно. Так как числа $n$ и 4 взаимно просты, то существуют числа $m \geqslant 4$ и $t \geqslant 1$ такие, что $11+4 m=|W U|=t n$.

Построим слово $T$, соответствуюшее данному $m$, и возьмем слово $\phi^{s}(T)$. В силу предложений $1.1,1.2$ слово $\phi^{s}(T)$ удовлетворяет свойству $(*)$. Обозначим через $S$ префикс $\phi^{s}(T)$ длины $t k$. Имеем

$$
\operatorname{lexp}(S)=\exp (S)=\frac{|S|}{\left|\phi^{s}(W U)\right|}=\frac{t k}{2^{s} t n}=\beta
$$

Покажем, что при подходяшем (достаточно большом) натуральном $q$ слово $\phi^{q}(S)$ заведомо можно достроить до $Z$-слова с локальной экспонентой $\beta$.

Заметим, что $\exp \left(1_{\phi^{s+q}(U)} \phi^{q}(S)\right)>\beta$. Напомним также, что последние буквы слова и его $\phi$-образа всегда различны; следовательно, $1_{\phi^{s+q}(U)}=1_{U}=b$ при четном $(s+q)$ и $1_{\phi^{s+q}(U)}=a$, если $(s+q)$ нечетно. Пусть $Q$ - произвольное слово такое, что $1_{Q}=a$. Пусть также $p$ - период некоторого собственного префикса $S$, причем $p<\operatorname{per}(S)$, а $P$ - максимальный префикс $S$ такой, что $\operatorname{per}(P)=p$. Тогда $\exp (P) \leqslant 7 / 3<\beta$ по свойству $(*)$. Следовательно, начиная с некоторого $q$, вьполняется неравенство

$$
\frac{2^{q}|P|+|Q|}{2^{q} p} \leqslant \frac{7}{3}+\frac{|Q|}{2^{q} p}<\beta .
$$


Применив предложение 1.1 , получаем $\exp \left(\phi^{q}(P)\right)=\exp (P)$ и, таким образом, $\exp \left(Q \phi^{q}(P)\right) \leqslant \beta$.

Нетрудно заметить, что слово $\phi^{q}(P)$ является максимальньм префиксом в $\phi^{q}(S)$ с периодом $2^{q} p$ (в силу того же предложения 1.1). Теперь выберем $q>0$ достаточно большим, чтобы оно подходило для любого $p$, являюшегося периодом префикса слова $S$. Кроме того, сделаем его таким, чтобы $(s+q)$ было четным числом; тогда, как отмечалось вьше, $1_{\phi^{s+q}(U)}=b$, а значит, слово $a \phi^{q}(S)$ не имеет периода $2^{s+q}|W U|$. Учитьвая равенство $\phi^{q}\left(0_{S}\right)=\phi^{q}(a)=U_{q}$, мы получим, что

$$
\operatorname{lexp}\left(Q \phi^{q}(S)\right) \leqslant \beta \Leftrightarrow \operatorname{lexp}\left(Q U_{q}\right) \leqslant \beta .
$$

Возьмем слово $Q=a b b a a b a$. Проверка показывает, что $\operatorname{lexp}\left(Q U_{q}\right)=7 / 3$. (В самом деле, $q>0$, а следовательно, $Q U_{q}$ имеет ровно одно подслово аabaa, справа от которого стоит элемент $T M$. Таким образом, если $P=X Y X Y X$ содержится в $Q U_{q}$, то aаbaa $\leqslant P$ и $|X Y|<5$. Отсюда $P=b a a b a a b$.) Следовательно, $\operatorname{lexp}\left(Q \phi^{q}(S)\right) \leqslant \beta$. Заметим еше, что слово $Q \phi^{q}(S)$ всегда имеет префикс $W$ :

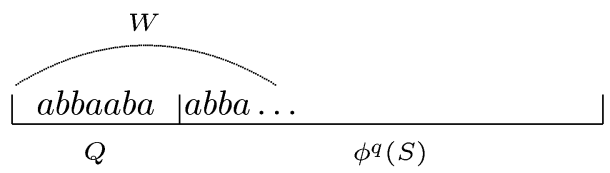

Проверка, аналогичная только что проведенной, показьвает, что $\operatorname{lexp}(\infty V W)=$ $7 / 3$. Заметив, что $\omega^{*}$-слово $\infty V$ и слово $\phi^{q}(S)$ равномерны, мы получаем, что на стыке слов $Q$ и $\phi^{q}(S)$ находится единственное подслово аabaa $\omega^{*}$-слова $\infty V Q \phi^{q}(S)$. Таким образом, $\operatorname{lexp}\left({ }_{\infty} V Q \phi^{q}(S)\right)=\beta$ :

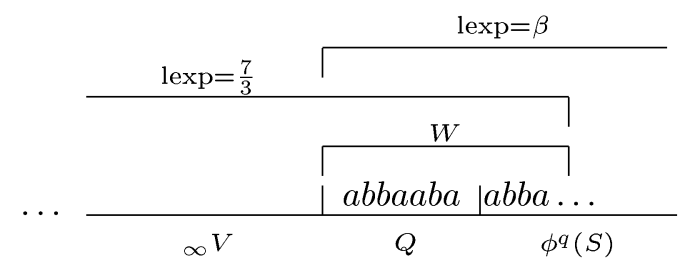

Для завершения построения $Z$-слова локальной экспоненты $\beta$ нам осталось произвести рассуждение для суффиксов слова $S$, аналогичное рассуждению, приведенному для префиксов. Без ограничения обшности мы можем считать, что $S b$ является префиксом $\phi^{s}(T)$. Тогда возьмем слово $Q^{\prime}$ такое, что $0_{Q^{\prime}}=a$, и найдем $q^{\prime}$, для которого равносильны условия $\operatorname{lexp}\left(\phi^{q^{\prime}}(S) Q\right) \leqslant \beta$ и $\operatorname{lexp}\left(\phi^{q^{\prime}}\left(1_{S}\right) Q\right) \leqslant \beta$. Выбрав $Q^{\prime}=a b a a b b a$, если $\phi^{q^{\prime}}(S)$ имеет суффикс $a b b a$, и $Q^{\prime}=W$, если этот суффикс равен $b a a b$, мы построим $\omega$-слово локальной экспоненты $\beta$, домножив слово $\phi^{q^{\prime}}(S) Q$ справа на $V_{\infty}$.

Пусть без ограничения общности $q \leqslant q^{\prime}$. Учитьвая, что $\phi^{q^{\prime}-q}(\infty V)=\infty V$, запишем $Z$-слово

$$
\mathbf{W}={ }_{\infty} V \phi^{q^{\prime}-q}(Q) \phi^{q^{\prime}}(S) Q^{\prime} V_{\infty} .
$$


Нам известно, что $\operatorname{lexp}\left(\infty V \phi^{q^{\prime}-q}(Q) \phi^{q^{\prime}}(S)\right)=\beta$ (по предложению 1.2) и $\operatorname{lexp}\left(\phi^{q^{\prime}}(S) Q^{\prime} V_{\infty}\right)=\beta$ по построению. Таким образом, если $\mathbf{W}$ содержит подслово $P$ экспоненты, большей $\beta$, то $\phi^{q^{\prime}}(S) \leqslant P$.

Напомним, что слово $\phi^{q^{\prime}}(S)$ является префиксом экспоненты $\beta$ в слове $\phi^{q^{\prime}+s}(T)$. В свою очередь, слово $T$ равно $(W U)^{3}$ по определению, причем по построению $|W U| \geqslant 27$ (мы выбирали $m \geqslant 4$, откуда длина слова $U$, состоящего из $m 2$-цепочек, не меньше 16). Рассмотрим слово $(W U)^{2} a b b a a b a a$. Согласно (i) оно не имеет периодов, промежуточных между $|W U|$ и $2|W U|$. Экспонента данного слова равна $2+8 /|W U|$, что меньше, чем $7 / 3$, а значит, меньше $\beta$. Тогда слово $\phi^{q^{\prime}+s}\left((W U)^{2} a b b a a b a a\right)$ является префиксом в $\phi^{q^{\prime}}(S)$. Пользуясь предложениями 1.1 и 1.2 , получаем, что слово $\phi^{q^{\prime}}(S)$ не имеет периодов, промежуточных между $2^{q^{\prime}+s}|W U|$ и $2^{q^{\prime}+s+1}|W U|$.

Первый из указанных периодов слово $P$ не может иметь по построению $\left(0_{Q^{\prime}}=a\right.$, а слово $\phi^{q^{\prime}}(S) a$ не имеет такого периода). Если же $P$ имеет период, не меньший второго из указанных, то его экспонента меньше двух: длина слова $\phi^{q^{\prime}-q}(Q) \phi^{q^{\prime}}(S) Q^{\prime}$, как нетрудно проверить, меньше двух таких периодов, при этом ни одно из слов $\phi^{q^{\prime}-q}(Q) \phi^{q^{\prime}}(S), \phi^{q^{\prime}}(S) Q^{\prime}$ не может иметь такого периода. (Это следует из предложений $1.1,1.2$ и уже неоднократно использовавшегося наблюдения, что слова $Q \phi^{q}(S)$ и $\phi^{q^{\prime}}(S) Q^{\prime}$ содержат только по одному подслову аabaa.) Таким образом, мы показали, что $Z$-слово $\mathbf{W}$ не содержит подслов экспоненты, большей $\beta$. Теорема доказана.

3.6. $Z$-слова с иррациональными экспонентами. Несмотря на то, что локальная экспонента $Z$-слова по определению является супремумом множества рациональных чисел, до сих пор все построенные нами $Z$-слова имели рациональную локальную экспоненту. Однако в доказательстве последней теоремы содержится идея, которая может быть использована для конструирования $Z$-слов с иррациональной локальной экспонентой. А именно, мы строили такое слово экспоненты $\beta$, к которому через специальные "переходные" слова можно присоединить $\omega$ и $\omega^{*}$-слово Туэ-Морса так, чтобы экспоненты всех подслов полученного $Z$-слова, не содержаших исходное слово экспоненты $\beta$, не превышали $7 / 3$.

Опираясь на конструкции, приведенные в доказательстве теоремы 3.3 , докажем следующий усиленный ее вариант.

Tеорема 3.8. Пусть $\gamma \in \mathbb{R}, 7 / 3<\gamma<3$. Тогда существует $Z$-слово $\mathbf{W}$ такое, что $\operatorname{lexp}(\mathbf{W})=\gamma$.

ДокАЗАТЕЛЬСТво. Возьмем последовательность рациональных чисел $\left\{\beta_{i}\right\}$, сходящуюся к $\gamma$ снизу. Без ограничения обшности предположим, что $\beta_{i+1}>\beta_{i}>$ $7 / 3$ для всех $i$. Для каждого $\beta_{i}$ построим $Z$-слово $\mathbf{W}_{i}$ локальной экспоненты $\beta_{i}$ с помощью конструкции, используемой в доказательстве теоремы 3.3. Главную часть этой конструкции составляет слово

$$
\phi^{q^{\prime}-q}(Q) \phi^{q^{\prime}}(S) Q^{\prime},
$$

или, двойственно,

$$
Q \phi^{q}(S) \phi^{q-q^{\prime}}\left(Q^{\prime}\right) .
$$

Обозначим данное слово через $S_{i}$. Конкретный вид слов $S, Q, Q^{\prime}$, приведенный в доказательстве теоремы 3.3 , нам не важен; напомним лишь, что слово $S_{i}$ содержит 
одно подслово аabaa, если $q \neq q^{\prime}$, и два таких подслова, если $q=q^{\prime}$. Так как на $q, q^{\prime}$ не накладывались никакие ограничения сверху, то одно из них всегда можно увеличить таким образом, чтобы выполнялось $q \neq q^{\prime}$. Поэтому мы будем считать, что $S_{i}$ содержит единственное подслово $a a b a a$.

Построим $Z$-слово искомой локальной экспоненты $\gamma$. Построение проведем по индукции. База индукции: пусть $W_{1}=S_{1} ; \operatorname{lexp}\left(W_{1}\right)=\beta_{1}$. Идею шага индукции вначале продемонстрируем на самом первом шаге.

Выберем такое четное положительное число $k_{1}$, что $\left|S_{1}\right|+\left|S_{2}\right| \leqslant 2^{k_{1}-1}$, и положим $W_{2}=S_{1} V_{k_{1}} S_{2}$, где $V_{k_{1}}$ - слово Туэ-Морса. Покажем, что $\operatorname{lexp}\left(W_{2}\right)=\beta_{2}$. В самом деле, слово $S_{1} V_{k_{1}}$ содержится в $\omega$-слове $S_{1} V_{\infty}$ локальной экспоненты $\beta_{1}$, а слово $V_{k_{1}} S_{2}$ содержится в $\omega^{*}$-слове $\infty V S_{2}$ локальной экспоненты $\beta_{2}$ (так как $k_{1}-$ четное число, слово $V_{k_{1}}$ является суффиксом в $\left.\infty V\right)$. Значит, если слово $W_{2}$ содержит подслово $P$ экспоненты, большей $\beta_{2}$, то $P$ содержит $V_{k_{1}}$. Так как $k_{1} \geqslant 2$, то $\operatorname{per}\left(V_{k_{1}}\right)=3 \cdot 2^{k_{1}-2}$ (см. п. $\left.1.1,4\right)$. Получаем

$$
\exp (P)=\frac{|P|}{\operatorname{per}(P)}<\frac{\left|W_{2}\right|}{\operatorname{per}\left(V_{k_{1}}\right)}=\frac{\left|S_{1}\right|+\left|S_{2}\right|+2^{k_{1}}}{3 \cdot 2^{k_{1}-2}} \leqslant \frac{2^{k_{1}-1}+2^{k_{1}}}{3 \cdot 2^{k_{1}-2}}=2,
$$

следовательно, локальная экспонента построенного слова действительно равна $\beta_{2}$.

Теперь рассмотрим шаг индукции в общем виде. Четное число $k_{n}$ выбираем так, чтобы

1) $\left|W_{n}\right|+\left|S_{n+1}\right| \leqslant 2^{k_{n}-1}$

2) $k_{n} \geqslant k_{n-1}+4$.

Положим $W_{n+1}=W_{n} V_{k_{n}} S_{n+1}$. Докажем, что $\operatorname{lexp}\left(W_{n+1}\right)=\beta_{n+1}$. В силу выбора $k_{n}$ имеем $\operatorname{lexp}\left(V_{k_{n}} S_{n+1}\right)=\beta_{n+1}$ и $\exp (P)<2$ для любого подслова $P$, содержащего $V_{k_{n}}$. Таким образом, достаточно показать, что $\operatorname{lexp}\left(W_{n} V_{k_{n}}\right)=\beta_{n}$. Данное слово имеет префикс $W_{n}$ локальной экспоненты $\beta_{n}$ (по предположению индукции) и суффикс $V_{k_{n-1}} S_{n} V_{k_{n}}$ той же локальной экспоненты (по построению). Указанные префикс и суффикс пересекаются в $W_{n} V_{k_{n}}$ по слову $V_{k_{n-1}} S_{n}$. Значит, подслово $P \leqslant W_{n} V_{k_{n}}$ может иметь экспоненту, большую $\beta_{n}$, только в случае $V_{k_{n-1}} S_{n} \leqslant P$. Это означает, в частности, что $P$ содержит подслово $a a b a a$. Если $\exp (P) \geqslant 2$, то число $\operatorname{per}(P)$ равно расстоянию между какими-нибудь двумя вхождениями $a a b a a$ в $P$. Но в силу выбора слова $V_{k_{n-1}}$ расстояние между подсловом $a a b a a$ из $S_{n}$ и подсловом $a a b a a$ из $S_{n-1}$ превосходит расстояние между любыми двумя такими подсловами, входящими в $W_{n-1}$. Таким образом, $P$ содержит ровно два подслова аabaа (входящие в $S_{n-1}$ и $S_{n}$ соответственно), т.е. $P=X a a b a a Y a a b a a Z$, где слова $X, Y$ и $Z$ равномерны. Имеем

$$
\operatorname{per}(P)>|Y|>\left|V_{k_{n-1}}\right|=2^{k_{n-1}}
$$

В то же время

$$
|X|<\left|S_{n-2} V_{k_{n-2}} S_{n-1}\right| \leqslant \frac{3}{2} \cdot\left|V_{k_{n-2}}\right|=\frac{3 \cdot 2^{k_{n-2}}}{2} \leqslant \frac{3 \cdot 2^{k_{n-1}}}{32}<\frac{\operatorname{per}(P)}{3} .
$$

Таким образом, $\exp (P)<7 / 3<\beta_{n}$. Мы доказали, что $\operatorname{lexp}\left(W_{n+1}\right)=\beta_{n+1}$.

Построенное по индукции $\omega$-слово будет иметь локальную экспоненту $\gamma$ по определению. Дописав к нему слева $\omega^{*}$-слово $\infty V$, мы и получим искомое $Z$-слово $\mathbf{W}$ :

$$
\mathbf{W}={ }_{\infty} V S_{1} V_{k_{1}} \ldots S_{i} V_{k_{i}} \ldots
$$


Теорема доказана.

Таким образом, мы показали, что $L_{\beta}^{Z} \subset L_{\beta}^{\prime Z}$ для всех $\beta \in[7 / 3,3)$. Это позволяет нам изобразить зависимости между $\beta$ и $L_{\beta}^{Z}$, между $\beta$ и $L_{\beta}^{\prime Z}$ в виде графиков, приведенных в п. 1.3.

Автор выражает глубокую благодарность участникам научных семинаров "Алгоритмические вопросы алгебры и логики" (МГУ, рук. член-корр. РАН С.И. Адян) и "Алгебраические системы" (УрГУ, рук. проф. Л.Н. Шеврин) за многочисленные ценные замечания.

\section{Список литературы}

1. Thue A. Über die gegenseitige Lage gleicher Teile gewisser Zeichenreihen // Skr. Vid. Kristiania I. Mat. Naturv. Klasse I. 1912. P. 1-67.

2. Арион C.E. Доказательство существования $n$-значных бесконечных асимметричных последовательностей // Матем. сб. 1937. Т. 2(44). № 4. С. 769-779.

3. Morse M., Hedlund G. A. Symbolic dynamics // Amer. J. Math. 1938. V. 60. P. 815-866.

4. Perrin D., Pin J.-E. Semigroups and automata on infinite words // Semigroups, Formal languages and Groups. Holland: Kluwer Acad. Publ., 1995. P. 49-72.

5. Gottschalk W. H., Hedlund G. A. Topological dynamics // Amer. Math. Soc. Colloq. Publ. 1955.

6. Branderburg F.-J. Uniformly growing $k$-th power free homomorphisms // Theor. Comput. Sci. 1983. V. 23. P. 69-82.

7. Kobayashi Y. Enumeration of irreducible binary words // Discr. Appl. Math. 1988. V. 20. P. 221-232.

8. Gottschalk W. H., Hedlund G. A. A characterization of the Morse minimal set // Proc. of Amer. Math. Soc. 1964. V. 15. P. 70-74.

9. Crochemore M., Goralčik P. Mutually avoidable ternary words of small exponent // Int. J. Alg. and Comp. 1991. V. 1. P. 407-410.

10. Shur A. M. Overlap-free words and Thue-Morse sequences // Int. J. Alg. and Comp. 1996. V. 6. P. 353-367.

11. Jezek J. Intervals in the lattice of varieties // Algebra Universalis. 1976. V. 6. P. 147-158.

12. Shur A. M. Binary words avoided by the Thue-Morse sequence // Semigroup Forum. 1996. V. 53. P. 212-219. 